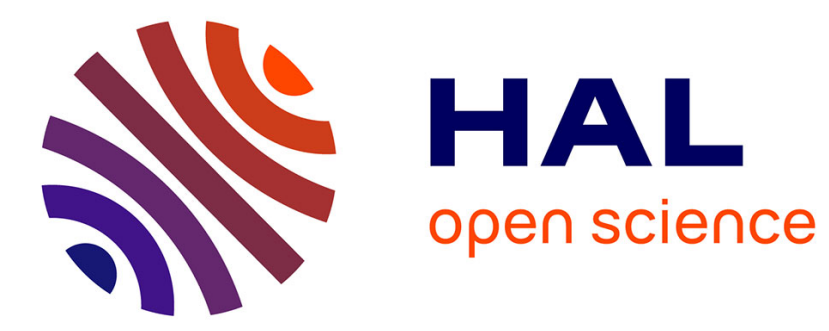

\title{
Inflation and bending of an orthotropic inflatable beam
}

\author{
Quang-Tung Nguyen, Jean-Christophe Thomas, Anh Le Van
}

\section{To cite this version:}

Quang-Tung Nguyen, Jean-Christophe Thomas, Anh Le Van. Inflation and bending of an orthotropic inflatable beam. Thin-Walled Structures, 2015, pp.129-144. 10.1016/j.tws.2014.11.015 . hal-01154692

\section{HAL Id: hal-01154692 \\ https://hal.science/hal-01154692}

Submitted on 22 May 2015

HAL is a multi-disciplinary open access archive for the deposit and dissemination of scientific research documents, whether they are published or not. The documents may come from teaching and research institutions in France or abroad, or from public or private research centers.
L'archive ouverte pluridisciplinaire HAL, est destinée au dépôt et à la diffusion de documents scientifiques de niveau recherche, publiés ou non, émanant des établissements d'enseignement et de recherche français ou étrangers, des laboratoires publics ou privés. 


\title{
INFLATION AND BENDING OF AN ORTHOTROPIC INFLATABLE BEAM
}

\author{
Quang-Tung NGUYEN ${ }^{\mathrm{a}}$, Jean-Christophe THOMAS ${ }^{\mathrm{b}}$, Anh LE VAN ${ }^{\mathrm{b}}$ \\ ${ }^{a}$ University of Danang, University of Science and Technology, \\ 54 Nguyen Luong Bang Street, Lien Chieu District, Da Nang, Vietnam \\ ${ }^{b}$ LUNAM Université, Université de Nantes-Ecole Centrale Nantes, \\ GeM (Institute for Research in Civil and Mechanical Engineering), CNRS UMR 6183 \\ 2, rue de la Houssinière, BP 92208, 44322 Nantes Cedex 3, France
}

\begin{abstract}
An inflatable beam is an airtight structure made of a soft technical fabric and subjected to an internal pressure which gives it a final cylindrical shape, a pre-stress in the membrane and a bearing capacity. Against all appearances, it is not a standard beam and it requires a specific formulation in order to take account of the internal pressure which plays a key role in its mechanical response.

This work deals with inflatable beams made of orthotropic materials. The first part of the paper is concerned with the inflation of the membrane tube, an important stage which is often neglected so far in the literature. As preliminaries of the bending problem studied in the next part of the paper, the constitutive law related to the inflated state of the tube - not the natural state - is investigated. It will be shown that the constitutive law related to the inflated pre-stressed state is not the same as the constitutive law related to the natural state. Expressions of the material coefficients involved in the former constitutive law will be established from the material coefficients defined on the natural reference configuration which are the only ones supposed to be known. The second part of the paper deals with the bending of the inflatable beam. The Timoshenko beam kinematics will be chosen because of the significant shear effect in the tube wall and the problem will be formulated in finite deformations in order to accounts for all the nonlinear effects, in particular the action due to the internal pressure which is a follower load. The nonlinear system of equations obtained will then be linearized around the pre-stressed configuration and will result in a more tractable linear system.
\end{abstract}


The proposed formulation allows a comprehensive study of the influence of the internal pressure on the geometry and material properties of orthotropic inflatable beams The analytical results will be compared with numerical results obtained from a nonlinear membrane finite element code.

Key words: Inflatable beam; membrane tube; orthotropy; inflation; bending

\section{INTRODUCTION}

Inflatable structures made of modern textiles have been used for several decades. Among their applications in space industry, there are deployable antennas, inflatable reentry capsules and inflatable solar power sails, mainly made of isotropic fabrics. The inflatable technology is also widely applied to terrestrial structures, both in civilian and military fields. Today, large inflatable structures are built for sports centres, exhibition halls and storage shelters. The fabrics used in these types of structures are often made of warp and weft threads which ensure the mechanical resistance and a coating which ensures the airtightness. In comparison with conventional structures, the inflatable structures may present some advantages in some specific cases: they are light, easily foldable, easily to transport, deploy. Moreover, it is not very expensive to manufacture them and keep them deployed.

The pressurized membrane structures are often made of bearing components which take the shape of tubes or beams. Each component is made of an airtight fabric and subjected to an internal pressure which gives it a final cylindrical shape, a pre-stress in the membrane and a bearing capacity. In the sequel, mention will be made of two types of reference configuration:

(i) the natural configuration, where there is no external loads and the stress field is zero. The geometry and material properties are supposed to be known in the natural configuration. The membrane has no bearing capacity in this state;

(ii) the pre-stressed configuration, where the membrane structure is subjected to the internal pressure only. The geometry and, as will be seen below, the material properties are different from those in the natural state. The bearing capacity of the structure should increase with the pre-stress, i.e. with the internal pressure. 
Several works have been conducted in the literature to predict the behavior of an inflated tube subjected to bending loads. In these works, the reference configuration corresponds to the pre-stressed configuration of the inflated tube.

The earliest analytical expressions for the load-deflection response and the collapse load of a cantilever pressurized membrane tube can be found in Comer and Levy's paper [1]. In this work, the usual Euler-Bernoulli kinematics was chosen and it was assumed that the tube is made of an isotropic linear elastic material. Later, Webber [2] extended Comer and Levy's theory [1] to the case of a tube subjected to bending and twisting, and succeeded in determining how a twisting moment modifies the deflection, the wrinkling load as well as the collapse load. Afterwards, Main et al. [3] improved Comer and Levy's theory of pressurized membrane tubes [1] by considering the effect of the bi-axial stress state on the wrinkling. Experiments were conducted on pressurized tubes with circular cross-sections and the results obtained were compared with those given by Comer and Levy's theory [1]. In another study, Main [4] took the orthotropic property of the fabric into account so as to enhance his previously developed theory. Suhey et al. [5] carried out numerical computations on anisotropic pressurized membrane tubes by means of membrane finite elements and validated their finite element model by comparing their numerical results with Main et al.'s theoretical results in [3],[4].

When using the Euler-Bernoulli kinematics, the internal pressure does not appear in the expression of the deflection. In order to improve the previous formulations, many other authors preferred to use the Timoshenko kinematics which is more appropriate for thin-walled beams.

A major contribution in this formulation type is due to Fichter [6] who developed a theory for pressurized cylindrical membrane tubes made of isotropic membrane. His approach was based on the minimization of the total potential energy. After linearization, Fichter successfully derived the analytical equations for the bending problem of a pressurized membrane tube, exhibiting the very term of the internal pressure in the deflection expression.

Steeves [7] adopted a similar approach and proposed solutions in terms of Green functions. Wielgosz and Thomas [8] [9] dealt with analytical solutions for inflatable 
isotropic beams and panels by establishing the equilibrium equations in the deformed state in order to incorporate the internal pressure in their formulation. They considered the internal pressure as a follower force which represents the strengthening effect on the bending and shear stiffnesses. Le van and Wielgosz [10] improved Fichter's theory [6] by using the virtual power principle in the context of the total Lagrangian formulation. They considered large displacements and rotations in order to take account of all the nonlinear terms in the kinematic and equilibrium equations, and proposed solutions for the bending and buckling of a pressurized isotropic membrane tube. Davids and Zhang [11] confirmed Fichter's results by considering the pressure work during the volume change in isotropic Timoshenko beams. On the basis of [10], Apedo et al. [12], Nguyen et al. [13] went further by developing a theory for pressurized membrane tube made of an orthotropic material. They first used a 3D kinematics and then linearized their formulation. The bending and buckling problems were investigated, and the results obtained show that it is essential to consider the fabric orthotropy in the computations.

In all the above-mentioned works, the reference configuration is the pre-stressed configuration, yet the material coefficients used in the constitutive law are those related to the natural configuration, which corresponds to the tube without internal pressure. On the other hand, studies on the behavior of fabrics used in pressurized membrane structures have shown the fabric characteristics in the pre-stressed state differ from those in the natural state and depend on the internal pressure.

Cavallaro et al. [14] utilized finite element analysis to compute the load-displacement curve of a pressurized membrane tube subjected to four-point bending conditions and found that the mechanical response strongly depends on the internal pressure. Turner et al. [15] conducted torsion tests on pressurized membrane tubes subjected to different internal pressures with the aim of determining the shear modulus of the fabric in the pre-stressed configuration. Their experimental results also reveal that the shear modulus depends on the internal pressure and the larger the pressure is, the larger the shear modulus. Later on, Davids and Zhang [11] realized four-point bending tests with the same fabric as in [15] and with different internal pressures. By taking the shear moduli in [15], these authors performed an inverse analysis upon the load-displacement and obtained the Young's modulus. They noticed that the Young's modulus of the 
fabric in the pre-stressed configuration increases with the internal pressure. A beam finite element was designed to compute the load-displacement response of the pressurized membrane tube and the input data used were the material coefficients in the pre-stressed configuration of the structure. A good correlation found between the numerical model and the experimental deflection indicated that it is essential to use the material coefficients related to the pre-stressed configuration. More recently, Kabche et al. [16] presented a complete procedure based on Turner et al.'s test [15] in order to quantify the fabric properties in the pre-stressed configuration, which are strongly dependent on the internal pressure. They also realized the same four-point bending test as Davids and Zhang [11] and made similar observations on the material coefficients related to the pre-stressed configuration.

Inflatable structures are usually made of coated woven fabrics, which mostly display an anisotropic and viscoelastic behavior. In most of the above-mentioned studies, coated fabrics are modeled - as commonly practiced in the field of tensile structures - as orthotropic membranes under the plane stress assumption. Accordingly, we will not deal here with fabrics but with orthotropic membranes and we will assume that the membrane obeys the Saint Venant - Kirchhoff orthotropic elastic law. Elaborate models of coated fabrics and comparison with experimental results are much more complex subjects and they will not be considered in this paper. Our aim is to propose a comprehensive analytical model for orthotropic inflatable beams including both the inflation and the bending stages. The paper is organized as follows:

- Section 2 defines the problem of a pressurized membrane tube and points out that one should consider two distinct successive stages. The first is the inflation of the membrane tube, an important stage which is often neglected so far in the literature. The second stage is the bending of the pressurized membrane tube and it will be seen further that the results of this stage strongly depend on those acquired in the first stage.

- Sections 3 to 5 are concerned with the inflation of the membrane tube. Section 3 is a brief reminder of the analytical results given in [17] for the geometry of the inflated tube. As preliminaries of the bending problem studied in the next part 
of the paper, the constitutive law related to the inflated state of the tube - not the natural state - is investigated in Section 4. It will be shown that the tube remains orthotropic with respect to the inflated state. However, the material coefficients involved in the constitutive law are no longer the same. As far as the authors' knowledge, this is the first time a bridge is established between the material coefficients at the natural state and those at the pressurized state. The change of the material coefficients are numerically computed on different geometries of the tube and material properties of the membrane in Section 5. The values of the material coefficients for the membrane will be chosen of the same order as the coefficients found in the literature for fabrics. The numerical results obtained will be used in the bending stage of the tube.

- The last part of the paper - Sections 6 to 9 -deals with the bending of the tube. As will be described in Section 6, the Timoshenko beam kinematics is chosen because of the significant shear effect in the tube wall and the problem is formulated in finite deformations in order to accounts for all the nonlinear effects, in particular the action of the internal pressure which is a follower load. The result of this is a nonlinear system of equations governing the bending problem, which highlights the significant role of the internal pressure in the response of inflatable beams. In the next Section 7, the nonlinear system of equations obtained will be linearized around the pre-stressed configuration and will result in a more tractable linear system. Finally, Sections 8 and 9 constitute an application of the obtained analytical results to a cantilever inflatable beam. The numerical results for different tube geometries, materials and internal pressures will be compared with numerical results obtained from a nonlinear membrane finite element code.

\section{THE PROBLEM OF THE PRESSURIZED MEMBRANE TUBE}

The problem will be described using a kinematical time $t$ which is not necessarily the physical time as one is in Statics, and which is useful, however, to relate the successive states of the mechanical system. When studying the bending of a pressurized 
membrane tube, one has to consider two successive stages, formulated in two different ways and based on two different reference configurations (see 1).

1. The inflation stage. It is assumed that there exists a time $t_{\varnothing}$ when no external loading is applied on the tube and the stress field therein is zero. At that time, the tube is said in the stress-free (or natural) configuration (or state).

The typical feature of a membrane tube is that the tube has no stiffness in the absence of external loading and the only way for it to acquire a stiffness is to subject it to an internal pressure. Thus, the inflation is essential to membrane structures and deserves a separate study on its own, where the only loading is the internal pressure. The inflation stage will be formulated as a three-dimensional problem of nonlinear elasticity. The reference configuration will be chosen as the stress-free one and referred to as the natural reference configuration.

2. The bending stage. The internal pressure causes pre-tensions in the tube wall and enables the tube to bear other external loads. From a given time $t_{0}>t_{\varnothing}$, the internal pressure is kept fixed and other loads are then applied on the external surface in order to bend the tube.

The bending problem will be formulated using a Timoshenko kinematics with finite displacements and rotations. This time, the reference configuration is chosen equal to the one at time $t_{0}$, when the tube is subjected to the internal pressure only. The new reference configuration - where the stress field is not zero - is called the (pressurized) pre-stressed reference configuration. As for the final configuration, it is taken as the one at current time $t>t_{0}$ when the tube is both pressurized and bent.

Thus, one has to deal with two distinct reference configurations successively: one is stress-free and the other pre-stressed. Of course, quantities related to the pre-stressed reference configuration are not independent of quantities related to the stress-free reference configuration, there exist relations between them which will be established later. Let us now specify the notational convention adopted in this work.

a. If the reference configuration is pre-stressed, we shall adopt the standard notational convention for finite deformation problems. In general, a Lagrangian 

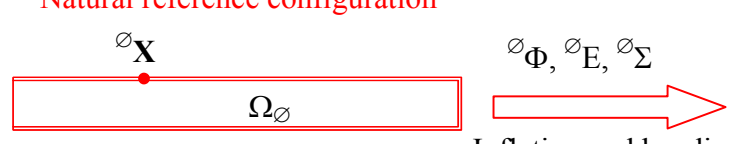

Inflation and bending

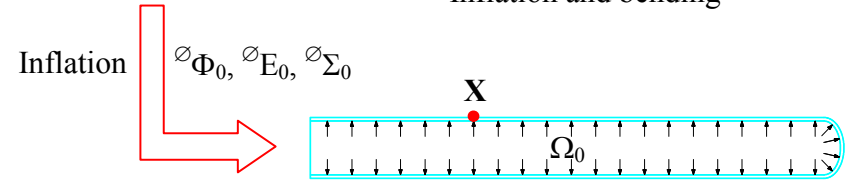

Pre-stressed reference configuration
Pressurized and bent configuration $\mathbf{x}$

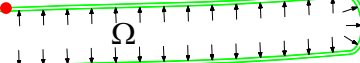

$\Phi, \mathrm{E}, \Sigma$

Figure 1: The three configurations of the membrane tube: the natural configuration, the pressurized prestressed configuration and the pressurized and bent configuration.

quantity related to the pre-stressed reference configuration will be denoted with an upper case letter, whereas its Eulerian counterpart related to the deformed configuration will be denoted with the same letter but in lower case. If the upper/lower case mode is not possible, the Lagrangian quantity will be written with a letter indexed by 0 , while its Eulerian counterpart will be written with the same letter without index. Accordingly, we denote (Figure 1):

- $\Omega_{0}$ the pressurized pre-stressed position, where the tube is subjected to the internal pressure only, $\Omega$ the current position, where the tube is both pressurized and bent;

- $\mathbf{X}$ the position of a current particle of the tube in the pressurized prestressed reference configuration and $\mathbf{x}$ the position of the same particle in the deformed configuration. These positions are related by

$$
\mathbf{x}=\mathbf{\Phi}(\mathbf{X}, t)
$$

where $\boldsymbol{\Phi}$ is the deformation from $\Omega_{0}$ to $\Omega$;

- $\boldsymbol{\sigma}(\mathbf{x}, t)$ the Cauchy stress tensor, $\boldsymbol{\Sigma}(\mathbf{X}, t)$ the second Piola-Kirchhoff stress tensor related to the pre-stressed state. The pre-stress in the tube solely subjected to the internal pressure then is $\boldsymbol{\Sigma}_{0}(\mathbf{X}) \equiv \boldsymbol{\Sigma}\left(\mathbf{X}, t_{0}\right)$.

b. As mentioned above, the reference configuration which is obvious in the inflation stage of the tube is the stress-free configuration, not the pre-stressed one. In order 
to distinguish the two reference configurations, we shall either attach the index $\varnothing$ to any quantity related to the stress-free configuration, or write the index $\varnothing$ instead of index 0. Accordingly, we denote (Figure 1):

- $\Omega_{\varnothing}$ the stress-free reference position;

- ${ }^{\varnothing} \mathbf{X}$ the position of a current particle of the tube in the stress-free configuration. The current position $\mathbf{x}$ of the same particle is related to ${ }^{\varnothing} \mathbf{X}$ by

$$
\mathbf{x}={ }^{\varnothing} \boldsymbol{\Phi}\left({ }^{\varnothing} \mathbf{X}, t\right)
$$

where ${ }^{\varnothing} \boldsymbol{\Phi}$ is the deformation from $\Omega_{\varnothing}$ to $\Omega$;

$-{ }^{\varnothing} \boldsymbol{\Sigma}\left({ }^{\varnothing} \mathbf{X}, t\right)$ the second Piola-Kirchhoff stress tenssor related to the stressfree state.

From (1) and (2), the two deformations $\boldsymbol{\Phi}$ and ${ }^{\varnothing} \boldsymbol{\Phi}$ are not independent but related by

$$
\mathbf{x}=\boldsymbol{\Phi}(\mathbf{X}, t)={ }^{\varnothing} \boldsymbol{\Phi}\left({ }^{\varnothing} \mathbf{X}, t\right)
$$

Making $t=t_{0}$ in (3) gives

$$
\mathbf{X}=\boldsymbol{\Phi}\left(\mathbf{X}, t_{0}\right)={ }^{\varnothing} \boldsymbol{\Phi}\left({ }^{\varnothing} \mathbf{X}, t_{0}\right) \equiv{ }^{\varnothing} \boldsymbol{\Phi}_{0}\left({ }^{\varnothing} \mathbf{X}\right)
$$

The inflation of the tube is defined by the deformation ${ }^{\varnothing} \boldsymbol{\Phi}_{0}$ from the stress-free configuration $\Omega_{\varnothing}$ to the pressurized pre-stressed configuration $\Omega_{0}$.

Let $\left(\mathbf{e}_{x}, \mathbf{e}_{y}, \mathbf{e}_{z}\right)$ be a fixed Cartesian basis. It is assumed that the tube in its stressfree reference state is cylindrical of axis $O \mathbf{e}_{x}$, thickness $H_{\varnothing}$, radius $R_{\varnothing}$ and length $L_{\varnothing}$, Moreover, the tube is made of an orthotropic material with the orthotropy directions $\mathbf{e}_{\ell}, \mathbf{e}_{t}$ in the reference configuration parallel to the tube axis and the tube circumference, respectively, as shown in Figure 2 (there is no possible confusion of index $t$ denoting the tangential orthotropy direction with above-mentioned time $t$ ). Thus, the third orthotropy direction is radial. The local orthotropy basis $\left(\mathbf{e}_{\ell}, \mathbf{e}_{t}, \mathbf{e}_{n}\right)$ is related to the Cartesian basis by $\mathbf{e}_{\ell}=\mathbf{e}_{x}, \mathbf{e}_{t}=\mathbf{e}_{y} \cos \varphi+\mathbf{e}_{z} \sin \varphi, \mathbf{e}_{n}=-\mathbf{e}_{y} \sin \varphi+\mathbf{e}_{z} \cos \varphi$, where $\varphi$ designates the angle from $\mathbf{e}_{y}$ to $\mathbf{e}_{t}$.

The goal of the paper is to study the bending of the pressurized tube and answer the following question: 

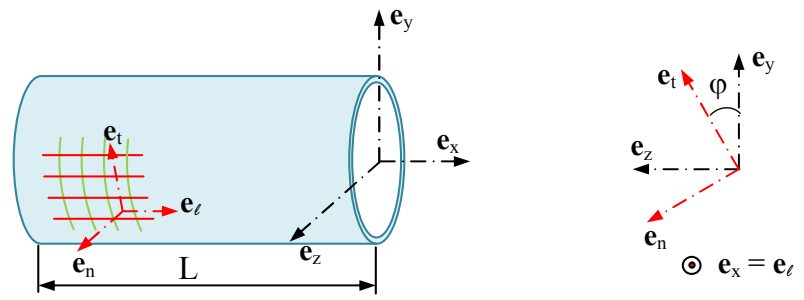

Figure 2: Local orthotropy basis and fixed Cartesian basis

- When the pressurized membrane tube is bent, what are the expressions for the deflection and the cross-section rotation?

The answer to this question will be given in Sections 6 et seqq. For this purpose, the two following questions need to be answered beforehand:

- What is the geometry of the pressurized tube?

It is essential to precisely determine the length and the radius of the tube in the pressurized state, since the radius of a thin-walled structure has a significant influence on the cross-section area and the second moment of area and as a result, the tube radius and length have a signifiant influence on the deflection and the rotation. This is true for a standard thin-walled (not membrane) structure and as will be seen in Section 6, this is also true for the pressurized membrane tube.

Determining the geometry of the pressurized tube is a preliminary work which is independent of the bending study itself. The results have been obtained in [17], they will be briefly recalled in Section 3 with notations adapted to the present framework.

- What is the constitutive law related to the pressurized pre-stressed reference configuration?

Since the bending problem is formulated with respect to the pressurized prestressed reference configuration, the constitutive law employed must be a relationship between variables related to the pre-stress configuration. One has to 
derive this constitutive law from that related to the natural reference configuration, which is the only one supposed to be known.

This issue will be investigated in Section 4.

\section{GEOMETRY OF THE INFLATED TUBE}

Let us begin with the the problem of the orthotropic membrane tube subjected to the internal pressure only. The reference configuration is chosen identical to the natural one $\Omega_{\varnothing}$, the final configuration is the pressurized pre-stressed one $\Omega_{0}$, and the transition from the former to the latter is described by the deformation ${ }^{\varnothing} \boldsymbol{\Phi}_{0}$ defined in (4), see Figure 1.

Let $p$ be the prescribed internal pressure. Owing to the axial symmetry of the problem, it can be assumed that the inflated tube remains cylindrical of axis $O \mathbf{e}_{x}$, and we denote $H, R, L$ the thickness, radius and length of the inflated tube, respectively.

The matrix of the deformation gradient tensor ${ }^{\varnothing} \mathbf{F}_{0} \equiv \frac{\partial^{\varnothing} \boldsymbol{\Phi}_{0}\left({ }^{\varnothing} \mathbf{X}\right)}{\partial^{\varnothing} \mathbf{X}}$ in the local orthotropy basis is expressed as

$$
\operatorname{Mat}\left({ }^{\varnothing} \mathbf{F}_{0} ; \mathbf{e}_{\ell}, \mathbf{e}_{t}, \mathbf{e}_{n}\right)=\left[\begin{array}{ccc}
\frac{L}{L_{\varnothing}} & 0 & 0 \\
0 & \frac{R}{R_{\varnothing}} & 0 \\
0 & 0 & \frac{H}{H_{\varnothing}}
\end{array}\right] \quad \text { hence }{ }^{\varnothing} J_{0} \equiv \operatorname{det}^{\varnothing} \mathbf{F}_{0}=\frac{L}{L_{\varnothing}} \frac{R}{R_{\varnothing}} \frac{H}{H_{\varnothing}}
$$

It is assumed that the constitutive law of the orthotropic membrane, related to the natural reference configuration, is hyperelastic, of the St Venant-Kirchhoff type:

$$
{ }^{\varnothing} \mathbf{E}_{0}={ }^{\varnothing} \mathbb{C}:{ }^{\varnothing} \boldsymbol{\Sigma}_{0}
$$

where ${ }^{\varnothing} \mathbf{E}_{0}=\frac{1}{2}\left({ }^{\varnothing} \mathbf{F}_{0}^{T}{ }^{\varnothing} \mathbf{F}_{0}-\mathbf{I}\right)$ and ${ }^{\varnothing} \boldsymbol{\Sigma}_{0}\left({ }^{\varnothing} \mathbf{X}\right) \equiv{ }^{\varnothing} \boldsymbol{\Sigma}\left({ }^{\varnothing} \mathbf{X}, t_{0}\right)$ denote the Green strain tensor and the second Piola-Kirchhoff stress tensor, respectively, both related to the natural configuration and computed at the inflated configuration; $\mathbf{I}$ is the identity tensor and finally ${ }^{\varnothing} \mathbb{C}$ is the compliance tensor related to the natural reference configuration.

The matrix of the compliance tensor ${ }^{\varnothing} \mathbb{C}$ in the orthotropy basis $\left(\mathbf{e}_{n}, \mathbf{e}_{\ell}, \mathbf{e}_{t}\right)$ is of the 
following form

$$
\operatorname{Mat}\left({ }^{\varnothing} \mathbb{C} ; \mathbf{e}_{\ell}, \mathbf{e}_{t}, \mathbf{e}_{n}\right)=\left[\begin{array}{ccc|ccc}
{ }^{\varnothing} C_{\ell \ell \ell \ell} & { }^{\varnothing} C_{\ell \ell t t} & { }^{\varnothing} C_{\ell \ell n n} & 0 & 0 & 0 \\
{ }^{\varnothing} C_{t t \ell \ell} & { }^{\varnothing} C_{t t t t} & { }^{\varnothing} C_{t t n n} & 0 & 0 & 0 \\
{ }^{\varnothing} C_{n n \ell \ell} & { }^{\varnothing} C_{n n t t} & { }^{\varnothing} C_{n n n n} & 0 & 0 & 0 \\
\hline 0 & 0 & 0 & { }^{\varnothing} C_{t n n t} & 0 & 0 \\
0 & 0 & 0 & 0 & { }^{\varnothing} C_{n \ell \ell n} & 0 \\
0 & 0 & 0 & 0 & 0 & { }^{\varnothing} C_{\ell t t \ell}
\end{array}\right]
$$

where the components of the matrix are functions of the Young's moduli ${ }^{\varnothing} E_{\ell},{ }^{\varnothing} E_{t}$, the Poisson's ratios ${ }^{\varnothing} v_{n \ell},{ }^{\varnothing} v_{t \ell},{ }^{\varnothing} v_{n t}$ and the shear moduli ${ }^{\varnothing} G_{\ell t},{ }^{\varnothing} G_{n \ell},{ }^{\varnothing} G_{t n}$ :

$$
\begin{array}{lll}
{ }^{\varnothing} C_{\ell \ell \ell \ell}=\frac{1}{{ }^{\varnothing} E_{\ell}} & { }^{\varnothing} C_{\ell \ell t t}={ }^{\varnothing} C_{t t \ell \ell}=-\frac{{ }^{\varnothing} v_{t \ell}}{{ }^{\varnothing} E_{t}} & { }^{\varnothing} C_{\ell \ell n n}={ }^{\varnothing} C_{n n \ell \ell}=-\frac{{ }^{\varnothing} v_{n \ell}}{{ }^{\varnothing} E_{n}} \\
{ }^{\varnothing} C_{t t t t}=\frac{1}{{ }^{\varnothing} E_{t}} & { }^{\varnothing} C_{t t n n}={ }^{\varnothing} C_{n n t t}=-\frac{{ }^{\varnothing} v_{n t}}{{ }^{\varnothing} E_{n}} & { }^{\varnothing} C_{n n n n}=\frac{1}{{ }^{\varnothing} E_{n}} \\
{ }^{\varnothing} C_{t n n t}=\frac{1}{{ }^{\varnothing} G_{t n}} & { }^{\varnothing} C_{n \ell \ell n}=\frac{1}{{ }^{\varnothing} G_{n \ell}} & { }^{\varnothing} C_{\ell t t \ell}=\frac{1}{{ }^{\varnothing} G_{\ell t}}
\end{array}
$$

The so formulated problem of the inflated tube was studied in [17] where it was shown that the length ratio $\frac{L}{L_{\varnothing}}$ satisfies the cubic equation

$$
\frac{H_{\varnothing}^{\varnothing} E_{\ell}}{p R_{\varnothing}}\left(\frac{L}{L_{\varnothing}}\right)^{3}+3{ }^{\varnothing} v_{\ell t}\left(\frac{L}{L_{\varnothing}}\right)^{2}-\left[\frac{H_{\varnothing}{ }^{\varnothing} E_{\ell}}{p R_{\varnothing}}+2 \frac{p R_{\varnothing}}{H_{\varnothing}{ }^{\varnothing} E_{t}}\left(1-{ }^{\varnothing} \boldsymbol{v}_{\ell t}{ }^{\varnothing} v_{t \ell}\right)\right] \frac{L}{L_{\varnothing}}-\left(1+{ }^{\varnothing} \boldsymbol{v}_{\ell t}\right)=0
$$

The input data are the tube geometry in the natural state: $L_{\varnothing}, R_{\varnothing}$, the material properties of the membrane in the natural state: ${ }^{\varnothing} E_{\ell} H_{\varnothing},{ }^{\varnothing} E_{t} H_{\varnothing},{ }^{\varnothing} v_{\ell t},{ }^{\varnothing} v_{t \ell}$, and the pressure $p$. Note that use has been made here of the products of the elasticity moduli by the membrane thickness, which actually are the material coefficients usually encountered in the study of textile membranes. The analytical expression for length $L$ in the inflated state is obtained by solving Equation (8) using Cardan's formula. The radius $R$ in the inflated state is then given by

$$
\left(\frac{R}{R_{\varnothing}}\right)^{2}=\frac{H_{\varnothing}^{\varnothing} E_{\ell}}{p R_{\varnothing}} \frac{L}{L_{\varnothing}}\left[\left(\frac{L}{L_{\varnothing}}\right)^{2}-1\right]+2{ }^{\varnothing} v_{\ell t}\left(\frac{L}{L_{\varnothing}}\right)^{2}
$$

\section{THE CONSTITUTIVE LAW RELATED TO THE PRESSURIZED PRE-STRESSED STATE}

In the previous study of the tube subjected to the internal pressure only, it is natural to make use of the orthotropic St Venant-Kirchhoff constitutive law related to the 
natural reference configuration. As regards the bending problem which will be investigated in the sequel, its analysis requires the constitutive law related to the pressurized pre-stressed configuration. This section describes how to derive such a law from the constitutive law related to the natural state, it will be shown that (i) the constitutive law related to the pre-stressed state remains orthotropic as in the natural state, (ii) however, the material coefficients related to the latter state are different from those in the former state.

\subsection{The constitutive law written in the orthotropy basis}

By means of (3) and (4), let us first establish the relationship between the deformation gradient tensors ${ }^{\varnothing} \mathbf{F} \equiv \frac{\partial^{\varnothing} \boldsymbol{\Phi}\left({ }^{\varnothing} \mathbf{X}\right)}{\partial^{\varnothing} \mathbf{X}}$ and $\mathbf{F}=\frac{\partial \Phi(\mathbf{X})}{\partial \mathbf{X}}$, respectively related to the natural and the pre-stressed states:

$$
{ }^{\varnothing} \mathbf{F} \equiv \frac{\partial^{\varnothing} \boldsymbol{\Phi}\left({ }^{\varnothing} \mathbf{X}\right)}{\partial^{\varnothing} \mathbf{X}}=\frac{\partial \Phi(\mathbf{X})}{\partial \mathbf{X}} \frac{\partial^{\varnothing} \boldsymbol{\Phi}_{0}\left({ }^{\varnothing} \mathbf{X}\right)}{\partial^{\varnothing} \mathbf{X}}
$$

or

$$
{ }^{\varnothing} \mathbf{F}=\mathbf{F}^{\varnothing} \mathbf{F}_{0} \quad \text { hence } \quad{ }^{\varnothing} J \equiv \operatorname{det}{ }^{\varnothing} \mathbf{F}=J{ }^{\varnothing} J_{0} \quad(J \equiv \operatorname{det} \mathbf{F})
$$

There follows the relation between the Green tensors ${ }^{\varnothing} \mathbf{E}=\frac{1}{2}\left({ }^{\varnothing} \mathbf{F}^{T \varnothing} \mathbf{F}-\mathbf{I}\right)$ and $\mathbf{E}=\frac{1}{2}\left(\mathbf{F}^{T} \mathbf{F}-\mathbf{I}\right)$, respectively related to the natural and the pre-stressed states:

$$
{ }^{\varnothing} \mathbf{E}-{ }^{\varnothing} \mathbf{E}_{0}=\frac{1}{2}\left({ }^{\varnothing} \mathbf{F}^{T} \varnothing \mathbf{F}-{ }^{\varnothing} \mathbf{F}_{0}^{T}{ }^{\varnothing} \mathbf{F}_{0}\right)=\frac{1}{2}\left({ }^{\varnothing} \mathbf{F}_{0}^{T} \mathbf{F}^{T} \mathbf{F}^{\varnothing} \mathbf{F}_{0}-{ }^{\varnothing} \mathbf{F}_{0}^{T}{ }^{\varnothing} \mathbf{F}_{0}\right)=\frac{1}{2} \varnothing \mathbf{F}_{0}^{T}\left(\mathbf{F}^{T} \mathbf{F}-\mathbf{I}\right){ }^{\varnothing} \mathbf{F}_{0}
$$

or

$$
{ }^{\varnothing} \mathbf{E}_{0}={ }^{\varnothing} \mathbf{E}-{ }^{\varnothing} \mathbf{F}_{0}^{T} \mathbf{E}^{\varnothing} \mathbf{F}_{0}
$$

Let us now derive the relation between the stress tensors related to the different configurations. The Cauchy stress tensor $\sigma(\mathbf{x})$ can either be expressed in terms of the Piola-Kirchhoff stress tensor ${ }^{\varnothing} \boldsymbol{\Sigma}\left({ }^{\varnothing} \mathbf{X}\right)$ related to the natural reference configuration, or in terms of $\boldsymbol{\Sigma}(\mathbf{X})$ related to the pre-stressed reference configuration:

$$
\boldsymbol{\sigma}=\frac{1}{{ } \boldsymbol{J}}{ }^{\varnothing} \mathbf{F}^{\varnothing} \boldsymbol{\Sigma}{ }^{\varnothing} \mathbf{F}^{T}=\frac{1}{J} \mathbf{F} \boldsymbol{\Sigma} \mathbf{F}^{T}
$$

Hence

$$
{ }^{\varnothing} \boldsymbol{\Sigma}=\frac{{ }^{\varnothing}}{J} \varnothing \mathbf{F}^{-1} \mathbf{F} \boldsymbol{\Sigma} \mathbf{F}^{T}{ }^{\varnothing} \mathbf{F}^{-T}
$$


The above relation can be simplified using (10) and one reaches the following relation between the Piola-Kirchhoff stress tensors $\boldsymbol{\Sigma}$ and ${ }^{\varnothing} \boldsymbol{\Sigma}$ :

$$
{ }^{\varnothing} \boldsymbol{\Sigma}={ }^{\varnothing} J_{0}^{\varnothing} \mathbf{F}_{0}^{-1} \boldsymbol{\Sigma}{ }^{\varnothing} \mathbf{F}_{0}^{-T}
$$

At time $t_{0}$, i.e. when there is no other loads applied on the tube than the pressure, the pressurized and bent configuration is identical to the pre-stressed reference configuration. Expression (12) then gives the relation between the pre-stress $\boldsymbol{\Sigma}_{0}(\mathbf{X})$ and the stress ${ }^{\varnothing} \boldsymbol{\Sigma}_{0}\left({ }^{\varnothing} \mathbf{X}\right)$ involved in (6):

$$
{ }^{\varnothing} \boldsymbol{\Sigma}_{0}={ }^{\varnothing} J_{0}{ }^{\varnothing} \mathbf{F}_{0}^{-1} \boldsymbol{\Sigma}_{0}{ }^{\varnothing} \mathbf{F}_{0}^{-T}
$$

Inserting (11) and (13) into the constitutive relation (6) related to the natural configuration yields

$$
{ }^{\varnothing} \mathbf{E}-{ }^{\varnothing} \mathbf{F}_{0}^{T} \mathbf{E}{ }^{\varnothing} \mathbf{F}_{0}={ }^{\varnothing} \mathbb{C}:\left({ }^{\varnothing} J_{0}{ }^{\varnothing} \mathbf{F}_{0}^{-1} \boldsymbol{\Sigma}_{0}{ }^{\varnothing} \mathbf{F}_{0}^{-T}\right)
$$

Therefore

$$
\begin{aligned}
{ }^{\varnothing} \mathbf{F}_{0}^{T} \mathbf{E}{ }^{\varnothing} \mathbf{F}_{0} & ={ }^{\varnothing} \mathbf{E}-{ }^{\varnothing} J_{0}{ }^{\varnothing} \mathbb{C}:\left({ }^{\varnothing} \mathbf{F}_{0}^{-1} \boldsymbol{\Sigma}_{0}{ }^{\varnothing} \mathbf{F}_{0}^{-T}\right) \\
\mathbf{E} & ={ }^{\varnothing} \mathbf{F}_{0}^{-T}\left({ }^{\varnothing} \mathbb{C}:{ }^{\varnothing} \boldsymbol{\Sigma}\right){ }^{\varnothing} \mathbf{F}_{0}^{-1}-{ }^{\varnothing} J_{0}{ }^{\varnothing} \mathbf{F}_{0}^{-T}\left[{ }^{\varnothing} \mathbb{C}:\left({ }^{\varnothing} \mathbf{F}_{0}^{-1} \boldsymbol{\Sigma}_{0}{ }^{\varnothing} \mathbf{F}_{0}^{-T}\right)\right]{ }^{\varnothing} \mathbf{F}_{0}^{-1} \\
& ={ }^{\varnothing} \boldsymbol{J}_{0}{ }^{\varnothing} \mathbf{F}_{0}^{-T}\left[{ }^{\varnothing} \mathbb{C}:\left({ }^{\varnothing} \mathbf{F}_{0}^{-1}\left(\boldsymbol{\Sigma}-\boldsymbol{\Sigma}_{0}\right){ }^{\varnothing} \mathbf{F}_{0}^{-T}\right)\right]{ }^{\varnothing} \mathbf{F}_{0}^{-1}
\end{aligned}
$$

or in components:

$\forall i, j, k, \ell \in\{\ell, t, n\}, \quad E_{i j}={ }^{\varnothing} J_{0}\left({ }^{\varnothing} \mathbf{F}_{0}^{-1}\right)_{m i}\left({ }^{\varnothing} \mathbf{F}_{0}^{-1}\right)_{n j}\left({ }^{\varnothing} \mathbf{F}_{0}^{-1}\right)_{p k}\left({ }^{\varnothing} \mathbf{F}_{0}^{-1}\right)_{q \ell}{ }^{\varnothing} C_{m n p q}\left(\Sigma-\Sigma_{0}\right)_{\ell k}$

where implicit summation is implied over repeated indices $m, n, p, q \in\{\ell, t, n\}$. Defining the 4 th-order tensor $\mathbb{C}$ as

$$
C_{i j k \ell}={ }^{\varnothing} J_{0}\left({ }^{\varnothing} \mathbf{F}_{0}^{-1}\right)_{m i}\left({ }^{\varnothing} \mathbf{F}_{0}^{-1}\right)_{n j}\left({ }^{\varnothing} \mathbf{F}_{0}^{-1}\right)_{p k}\left({ }^{\varnothing} \mathbf{F}_{0}^{-1}\right)_{q \ell}{ }^{\varnothing} C_{m n p q}
$$

one finds that the constitutive law related to the pre-stressed configuration is also of the St Venant-Kirchhoff type:

$$
E_{i j}=C_{i j k \ell}:\left(\Sigma-\Sigma_{0}\right)_{\ell k} \quad \text { ou } \quad \mathbf{E}=\mathbb{C}:\left(\boldsymbol{\Sigma}-\boldsymbol{\Sigma}_{0}\right)
$$


Note that definition (15) looks like that of the spatial elasticity tensor, see, for instance, [18] p123, but it is in fact different in meaning. Relations (15) and (16) are particularly significant since they highlight the fact that all the material coefficients are affected by the change of the reference state. Tensor $\mathbb{C}$ defined in (15) appears as the compliance tensor related to the pre-stressed reference configuration. It is linked to the compliance tensor ${ }^{\varnothing} \mathbb{C}$ related to the natural configuration via the deformation gradient tensor ${ }^{\varnothing} \mathbf{F}_{0}$ in (5) which expresses the geometry change from the natural to the inflated state.

Inserting (5) and (7) into (15) leads to the matrix of the compliance tensor $\mathbb{C}$ in the orthotropy basis $\left(\mathbf{e}_{\ell}, \mathbf{e}_{t}, \mathbf{e}_{n}\right)$ and enables one to write the constitutive law in this basis as follows:

$$
\left\{\begin{array}{c}
E_{\ell \ell} \\
E_{t t} \\
E_{n n} \\
2 E_{t n} \\
2 E_{n \ell} \\
2 E_{\ell t}
\end{array}\right\}=\left[\begin{array}{ccc|ccc}
C_{\ell \ell \ell \ell} & C_{\ell \ell t t} & C_{\ell \ell n n} & 0 & 0 & 0 \\
C_{t t \ell \ell} & C_{t t t t} & C_{t t n n} & 0 & 0 & 0 \\
C_{n n \ell \ell} & C_{n n t t} & C_{n n n n} & 0 & 0 & 0 \\
\hline 0 & 0 & 0 & C_{t n n t} & 0 & 0 \\
0 & 0 & 0 & 0 & C_{n \ell \ell n} & 0 \\
0 & 0 & 0 & 0 & 0 & C_{\ell t t \ell}
\end{array}\right]\left\{\begin{array}{c}
\Sigma_{\ell \ell}-\Sigma_{\ell \ell}^{0} \\
\Sigma_{t t}-\Sigma_{t t}^{0} \\
\Sigma_{n n}-\Sigma_{n n}^{0} \\
\Sigma_{t n}-\Sigma_{t n}^{0} \\
\Sigma_{n \ell}-\Sigma_{n \ell}^{0} \\
\Sigma_{\ell t}-\Sigma_{\ell t}^{0}
\end{array}\right\}
$$

It can easily checked that $C_{\ell \ell t t}=C_{t t \ell \ell}, C_{\ell \ell n n}=C_{n n \ell \ell}$ and $C_{t t n n}=C_{n n t t}$. This means that a material which is orthotropic with respect to the natural configuration remains orthotropic with respect to the pressurized pre-stressed configuration. Recall that the result has been obtained in the case when the orthotropy directions are parallel to the axial and circumferential directions of the tube.

\subsection{The constitutive law written in the fixed basis}

Let us now write the constitutive law (16) in the fixed Cartesian basis $\left(\mathbf{e}_{x}, \mathbf{e}_{y}, \mathbf{e}_{z}\right)$, by applying, onto (17), a change of basis from the local orthotropy basis to the Cartesian basis. Some simple algebraic calculations show that the compliance matrix in the fixed Cartesian basis takes a form slightly different from that in the local orthotropy basis:

$$
\left\{\begin{array}{c}
E_{X X} \\
E_{Y Y} \\
E_{Z Z} \\
2 E_{Y Z} \\
2 E_{Z X} \\
2 E_{X Y}
\end{array}\right\}=\left[\begin{array}{cccccc}
C_{X X X X} & C_{X X Y Y} & C_{X X Z Z} & C_{X X Z Y} & 0 & 0 \\
C_{Y Y X X} & C_{Y Y Y Y} & C_{Y Y Z Z} & C_{Y Y Z Y} & 0 & 0 \\
C_{Z Z X X} & C_{Z Z Y Y} & C_{Z Z Z Z} & C_{Z Z Z Y} & 0 & 0 \\
C_{Y Z X X} & C_{Y Z Y Y} & C_{Y Z Z Z} & C_{Y Z Z Y} & 0 & 0 \\
0 & 0 & 0 & 0 & C_{Z X X Z} & C_{Z X Y X} \\
0 & 0 & 0 & 0 & C_{X Y X Z} & C_{X Y Y X}
\end{array}\right]\left\{\begin{array}{c}
\Sigma_{X X}-\Sigma_{X X}^{0} \\
\Sigma_{Y Y}-\Sigma_{Y Y}^{0} \\
\Sigma_{Z Z}-\Sigma_{Z Z}^{0} \\
\Sigma_{Y Z}-\Sigma_{Y Z}^{0} \\
\Sigma_{Z X}-\Sigma_{Z X}^{0} \\
\Sigma_{X Y}-\Sigma_{X Y}^{0}
\end{array}\right\}
$$


The components of the compliance matrix in the Cartesian basis are, by denoting $c=\cos \varphi, s=\sin \varphi($ see angle $\varphi$ in Figure 2$)$ :

$$
\begin{aligned}
& C_{X X X X}=C_{\ell \ell \ell \ell} \\
& C_{X X Y Y}=C_{Y Y X X}=C_{\ell \ell t t} c^{2}+C_{\ell \ell n n} s^{2} \\
& C_{X X Z Z}=C_{Z Z X X}=C_{\ell \ell t t} s^{2}+C_{\ell \ell n n} c^{2} \\
& \begin{array}{l}
C_{X X Z Y}=C_{Y Z X X}=2\left(C_{\ell \ell t t}-C_{\ell \ell n n}\right) s c \\
C_{Y Y Y Y}=C_{t t t t} c^{4}+2 C_{t t n n} s^{2} c^{2}+C_{n n n n} s^{4}+C_{t n n t} s^{2} c^{2}
\end{array} \\
& C_{Y Y Z Z}=C_{Z Z Y Y}=\left(C_{t t t t}+C_{n n n n}\right) s^{2} c^{2}+C_{t t n n}\left(s^{4}+c^{4}\right)-C_{t n n t} s^{2} c^{2} \\
& C_{Y Y Z Y}=C_{Y Z Y Y}=2\left(C_{t t t t} c^{3} s-C_{n n n n} s^{3} c\right)+2 C_{t t n n}\left(s^{3} c-c^{3} s\right)-C_{t n n t}\left(c^{3} s-s^{3} c\right) \\
& C_{Z Z Z Z}=C_{t t t t} s^{4}+2 C_{t t n n} s^{2} c^{2}+C_{n n n n} c^{4}+C_{t n n t} s^{2} c^{2} \\
& C_{Z Z Z Y}=C_{Y Z Z Z}=2\left(C_{t t t t} s^{3} c-C_{n n n n} c^{3} s\right)-2 C_{t t n n}\left(s^{3} c-c^{3} s\right)+C_{t n n t}\left(c^{3} s-s^{3} c\right) \\
& C_{Y Z Z Y}=4\left(C_{t t t t}+C_{n n n n}-2 C_{t t n n}\right) s^{2} c^{2}+C_{t n n t}\left(c^{2}-s^{2}\right)^{2} \\
& C_{Z X X Z}=c^{2} C_{n \ell \ell n}+s^{2} C_{\ell t t \ell} \\
& C_{Z X Y X}=C_{X Y X Z}=\left(C_{\ell t t \ell}-C_{n \ell \ell n}\right) s c \\
& C_{X Y Y X}=s^{2} C_{n \ell \ell n}+c^{2} C_{\ell t t \ell}
\end{aligned}
$$

\subsection{Taking account of the plane stress assumption}

Let us denote $\boldsymbol{\Sigma}^{L}=\boldsymbol{\Sigma}-\boldsymbol{\Sigma}_{0}$ the part of the stress tensor which is linear in terms of the strain in the constitutive law (16) (here the superscript $L$ reminds of the linear part of the stress tensor, it should not be confused with the length $L$ of the inflated tube). From the mechanical point of view, tensor $\Sigma^{L}$ corresponds to the Piola-Kirchhoff stresses (related to the pre-stressed reference configuration) which are exclusively due to bending loads. It is assumed that $\Sigma^{L}$ satisfies the assumption of plane stress in the local orthotropy basis of the membrane:

$$
\operatorname{Mat}\left(\Sigma^{L} ; \mathbf{e}_{\ell}, \mathbf{e}_{t}, \mathbf{e}_{n}\right)=\left[\begin{array}{ccc}
\Sigma_{\ell \ell}^{L} & \Sigma_{\ell t}^{L} & 0 \\
\Sigma_{t \ell}^{L} & 0 & 0 \\
0 & 0 & 0
\end{array}\right]
$$

The assumption $\Sigma_{t t}^{L}=0$ means that, in bending, the tangential stress is negligible when compared with the axial and shear stresses. The components of $\Sigma^{L}$ in the orthotropy basis are expressed in terms of the components in the Cartesian basis by means of the change-of-basis matrix:

$$
\begin{array}{rlrl}
\Sigma_{\ell \ell}^{L} & =\Sigma_{X X}^{L} & \\
\Sigma_{t t}^{L} & =c^{2} \Sigma_{Y Y}^{L}+s^{2} \Sigma_{Z Z}^{L}+2 c s \Sigma_{Y Z}^{L} & & =0 \\
\Sigma_{n n}^{L} & =s^{2} \Sigma_{Y Y}^{L}+c^{2} \Sigma_{Z Z}^{L}-2 c s \Sigma_{Y Z}^{L} & & =0 \\
\Sigma_{t n}^{L} & =s c\left(\Sigma_{Z Z}^{L}-\Sigma_{Y Y}^{L}\right)+\left(c^{2}-s^{2}\right) \Sigma_{Y Z}^{L} & & =0 \\
\Sigma_{\ell n}^{L} & =c \Sigma_{X Z}^{L}-s \Sigma_{X Y}^{L} & & =0 \\
\Sigma_{\ell t}^{L} & =c \Sigma_{X Y}^{L}+s \Sigma_{X Z}^{L} &
\end{array}
$$


Equations $(21)_{2-4}$ give $\Sigma_{Y Y}^{L}=\Sigma_{Z Z}^{L}=\Sigma_{Y Z}^{L}=0$ and thus entail the following form of the matrix of $\Sigma^{L}$ in the fixed global basis:

$$
\operatorname{Mat}\left(\Sigma^{L} ; \mathbf{e}_{x}, \mathbf{e}_{y}, \mathbf{e}_{z}\right)=\left[\begin{array}{ccc}
\Sigma_{X X}^{L} & \Sigma_{X Y}^{L} & \Sigma_{X Z}^{L} \\
\Sigma_{Y X}^{L} & 0 & 0 \\
\Sigma_{Z X}^{L} & 0 & 0
\end{array}\right] \quad \text { with } \quad c \Sigma_{X Z}^{L}=s \Sigma_{X Y}^{L}
$$

Applying the antiplane form (22) to the constitutive law (18)-(19) gives

$$
\begin{array}{llll}
E_{X X} & =C_{X X X X} \Sigma_{X X}^{L} & & =C_{\ell \ell \ell \ell} \Sigma_{X X}^{L} \\
E_{Y Y} & =C_{Y Y X X} \Sigma_{X X}^{L} & & =\left(c^{2} C_{\ell \ell t t}+s^{2} C_{\ell \ell n n}\right) \Sigma_{X X}^{L} \\
E_{Z Z} & =C_{X X Z Z} \Sigma_{X X}^{L} & & =\left(s^{2} C_{\ell \ell t t}+c^{2} C_{\ell \ell n n}\right) \Sigma_{X X}^{L} \\
2 E_{Y Z} & =C_{Y Z X X} \Sigma_{X X}^{L} & & =2 s c\left(C_{\ell \ell t t}-C_{\ell \ell n n}\right) \Sigma_{X X}^{L} \\
2 E_{Z X} & =C_{Z X X Z} \Sigma_{Z X}^{L}+C_{Z X Y X} \Sigma_{X Y}^{L} & & =\left(c^{2} C_{n \ell \ell n}+s^{2} C_{\ell t t \ell}\right) \Sigma_{Z X}^{L}+s c\left(C_{\ell t t \ell}-C_{n \ell \ell n}\right) \Sigma_{X Y}^{L}=C_{\ell t t \ell} \Sigma_{Z X}^{L} \\
2 E_{X Y} & =C_{X Y X Z} \Sigma_{Z X}^{L}+C_{X Y Y X} \Sigma_{X Y}^{L} & & =s c\left(C_{\ell t t \ell}-C_{n \ell \ell n}\right) \Sigma_{Z X}^{L}+\left(s^{2} C_{n \ell \ell n}+c^{2} C_{\ell t t \ell}\right) \Sigma_{X Y}^{L}=C_{\ell t t \ell} \Sigma_{X Y}^{L}
\end{array}
$$

Among these relations, only the first and the last ones will be of use in the theory of pressurized membrane tubes. Let us define the Young's modulus $E_{\ell}$ along the axial direction of the tube and the shear modulus $G_{\ell t}$, in the pre-stressed reference configuration, as

$$
C_{\ell \ell \ell \ell}=\frac{1}{E_{\ell}} \quad C_{\ell t t \ell}=\frac{1}{G_{\ell t}}
$$

Relations $(23)_{1}$ and $(23)_{6}$ can be written as $\Sigma_{X X}^{L}=E_{\ell} E_{X X}$ and $\Sigma_{X Y}^{L}=2 G_{\ell t} E_{X Y}$, or

$$
\Sigma_{X X}=\Sigma_{X X}^{0}+E_{\ell} E_{X X} \quad \Sigma_{X Y}=\Sigma_{X Y}^{0}+2 G_{\ell t} E_{X Y}
$$

The moduli $E_{\ell}$ and $G_{\ell t}$ are related to their stress-free counterparts ${ }^{\varnothing} E_{\ell}$ and ${ }^{\varnothing} G_{\ell t}$ by (15) :

$$
E_{\ell} H=\left(\frac{L}{L_{\varnothing}}\right)^{3} \frac{R_{\varnothing}}{R} E_{\ell} H_{\varnothing} \quad G_{\ell t} H=\frac{L}{L_{\varnothing}} \frac{R}{R_{\varnothing}}{ }^{\varnothing} G_{\ell t} H_{\varnothing}
$$

These relationships clearly show how a geometry change due to the inflation modifies the material coefficients. One has only to experimentally determine the coefficients ${ }^{\varnothing} E_{\ell} H_{\varnothing},{ }^{\varnothing} G_{\ell t} H_{\varnothing}$ at the natural state, and it is then possible to derive the coefficients $E_{\ell} H, G_{\ell t} H$ for all sets of radius, length and pressure.

\section{NUMERICAL COMPUTATIONS}

In this section, numerical computations will be carried out in order to show the change in the geometry and the change in the material coefficients when one goes from the natural to the inflated configuration. 
Relations (25) show that the material coefficients $E_{\ell} H$ and $G_{\ell t} H$ in the pre-stressed configuration are related to their values in the natural configuration via the ratios $\frac{L}{L_{\varnothing}}$ and $\frac{R}{R_{\varnothing}}$. According to Relations (8) and (9), these ratios are themselves functions of radius $R_{\varnothing}$ of the stress-free tube, of the mechanical properties of the membrane in the natural state: ${ }^{\varnothing} E_{\ell} H_{\varnothing},{ }^{\varnothing} E_{t} H_{\varnothing},{ }^{\varnothing} v_{\ell t},{ }^{\varnothing} v_{t \ell}$, and of the pressure $p$. As these ratios are independent of $L_{\varnothing}$, we decide to perform the computations using only one value $L_{\varnothing}$ $(2.5 m)$ and three values for $R_{\varnothing}(0.1,0.15$ and $0.2 m)$. Thus, the computations will be done with three different tubes, whose characteristics are summarized in Table 1.

We consider one unbalanced orthotropic membrane, having two different elasticity moduli along the warp and weft directions. The membrane is rolled up around the tube axis in two different ways, giving rise to two different orientations which will be referred to in the sequel as Orientation 1 and Orientation 2:

- with Orientation 1, the warp is parallel to the tube axis;

- with Orientation 2, it is the weft which is parallel to the tube axis.

The values of the material coefficients are given in Table 1, they are of the same order as the coefficients found in the literature for fabrics (it also is the case for the tensile strength). To change from one orientation to another, one just has to invert the moduli ${ }^{\varnothing} E_{\ell} H_{\varnothing}$ and ${ }^{\varnothing} E_{t} H_{\varnothing}$ and the Poisson's ratios ${ }^{\varnothing} v_{\ell t}$ and ${ }^{\varnothing} v_{t \ell}$.

The internal pressure $p$ applied ranges from $50 \mathrm{kPa}$ from $600 \mathrm{kPa}$. The axial and hoop stresses $\sigma_{\ell \ell}^{0}$ and $\sigma_{t t}^{0}$ in the inflated tube must fulfil the tensile strength criterion, i.e. they must be less than a maximum stress $\sigma_{\max }$, which, for the considered membrane, is so chosen that $H \sigma_{\max }=360 \mathrm{daN} / 5 \mathrm{~cm}$. Since these stresses are related to the internal pressure $p$ by $H \sigma_{t t}^{0}=2 H \sigma_{\ell \ell}^{0}=p R$, the maximum pressure which can be prescribed on the tube is

$$
p_{\max }=\frac{H \sigma_{\max }}{R}
$$

The maximum value for the pressure depends on the radius $R$ in the inflated state. For the three tubes under consideration, of natural radius $R_{\varnothing}=0.1,0.15$ and $0.2 m$, it is found that the maximal internal pressures are about 600,450 and $300 \mathrm{kPa}$, respectively. In the sequel, all the curves will be drawn with internal pressure up to $600 \mathrm{kPa}$, and the 
portions of the curves beyond the maximal internal pressure will be shown by dashed lines.

Table 1: Data for the inflation of the orthotropic membrane tube

\begin{tabular}{lll}
\hline \hline TUBE GEOMETRY IN NATURAL CONFIGURATION & \\
Natural length $L_{\varnothing}: 2.5 \mathrm{~m}$ & \\
Natural radius $R_{\varnothing}: \quad 0.1 \mathrm{~m}, 0.15 \mathrm{~m}, 0.2 \mathrm{~m}$ & \\
\hline MATERIAL PROPERTIES IN NATURAL CONFIGURATION & & \\
& & \\
& & \\
Young's modulation 1 & Orientation 2 \\
Young's modulus in the axial direction ${ }^{\varnothing} E_{\ell} H_{\varnothing}$ & $600 \mathrm{kN} / \mathrm{m}$ & $300 \mathrm{kN} / \mathrm{m}$ \\
In-plane shear modulus ${ }^{\varnothing} G_{\ell t} H_{\varnothing}$ & $300 \mathrm{kN} / \mathrm{m}$ & $600 \mathrm{kN} / \mathrm{m}$ \\
Poisson's ratio ${ }^{\varnothing} v_{\ell t}$ & $12.5 \mathrm{kN} / \mathrm{m}$ & $12.5 \mathrm{kN} / \mathrm{m}$ \\
Poisson's ratio ${ }^{\varnothing} v_{t \ell}$ & 0.24 & 0.12 \\
Tensile strength & 0.12 & 0.24 \\
\hline LOADING & $360 \mathrm{daN} / 5 \mathrm{~cm}$ & $360 \mathrm{daN} / 5 \mathrm{~cm}$ \\
\hline Internal pressure $p: 50 \mathrm{kPa}$ to $600 \mathrm{kPa}$ & \\
\hline \hline
\end{tabular}

\subsection{Change of geometry versus the pressure}

For each given internal pressure $p$, application of formulas (8) and (9) gives the length $L$ and the radius $R$ of the inflated tube. The ratios $\frac{L}{L_{\varnothing}}$ and $\frac{R}{R_{\varnothing}}$ are displayed versus the internal pressure $p$ in Figures 3 and 4 for both membrane orientations.

In the case of Orientation 1 , the $\frac{L}{L_{\varnothing}}$ curves are clearly nonlinear whereas the $\frac{R}{R_{\varnothing}}$ curves appear to be nonlinear for larger pressures only. In the case of Orientation 2, the nonlinearity of both $\frac{L}{L_{\varnothing}}$ and $\frac{R}{R_{\varnothing}}$ curves can be observed for larger pressures only.

The nonlinearity is the more marked as the internal pressure $p$ is significant. Moreover, the larger the natural radius $R_{\varnothing}$ is, the more significantly $\frac{L}{L_{\varnothing}}$ and $\frac{R}{R_{\varnothing}}$ vary as functions of the internal pressure $p$.

\subsection{Moduli $E_{\ell} H$ and $G_{\ell t} H$ versus the pressure}

For each given internal pressure $p$, the deformed geometry of the tube is computed as described above and then use is made of the two Relations (25) in order to derive the material coefficients $E_{\ell} H$ and $G_{\ell t} H$ in the pressurized configuration. The variation of coefficients $E_{\ell} H, G_{\ell t} H$ versus pressure $p$ is displayed in Figures 5 and 6. It can be seen 


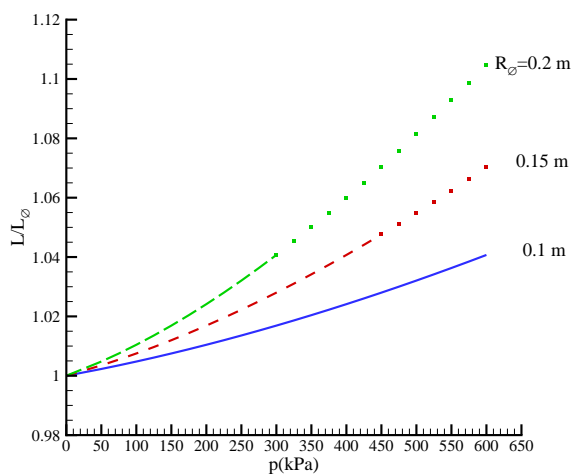

(a) Length variation

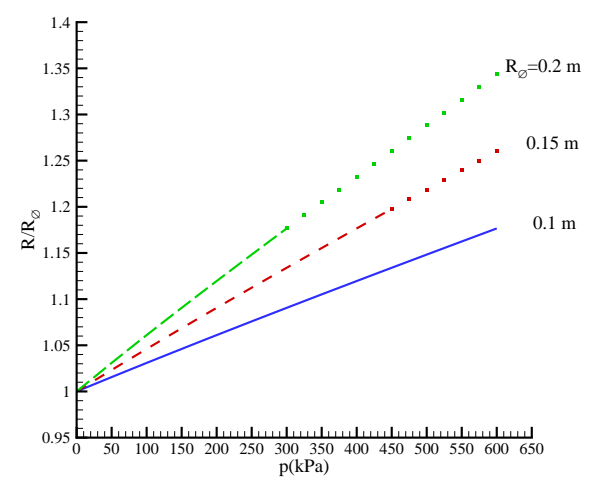

(b) Radius variation

Figure 3: Change of geometry versus the internal pressure -Orientation 1

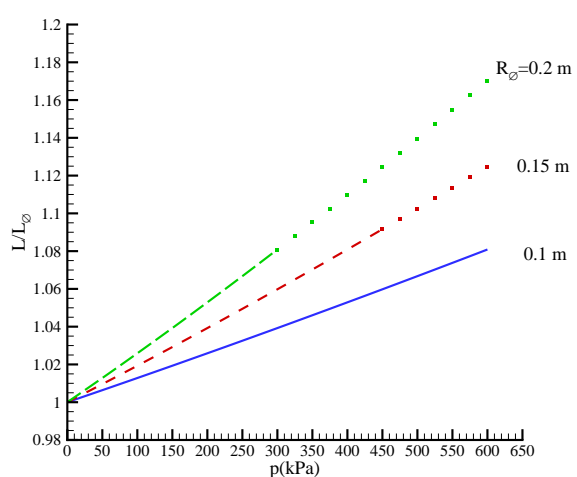

(a) Length variation

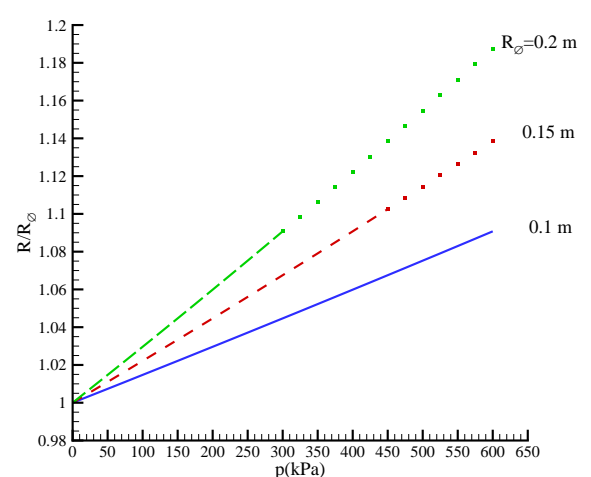

(b) Radius variation

Figure 4: Change of geometry versus the internal pressure - Orientation 2

that the larger the radius $R_{\varnothing}$ is, the more sensitive these coefficients are to the change of the internal pressure $p$, which is consistent with the above remark on the variations of $\frac{L}{L_{\varnothing}}$ and $\frac{R}{R_{\varnothing}}$.

\section{Orientation 1}

- Figure 5 related to the case of Orientation 1 shows that modulus $E_{\ell} H$ first decreases as the pressure $p$ increases, passes through a minimum and then in- 
creases.

- The pressure value $p$ corresponding to this minimum is all the smaller as $R_{\varnothing}$ is greater.

- The minimum value of $E_{\ell} H$ is exactly the same (575 $\mathrm{kNm}$ ) for three different radii $R_{\varnothing}$ and seems to be independent of the tube geometry in the natural reference configuration. The difference between the natural value ${ }^{\varnothing} E_{\ell} H_{\varnothing}$ and this minimum is $4.3 \%$.

- As regards the maximum pressure the tube can bear, it is found that, in the case of unbalanced membrane with ${ }^{\varnothing} E_{\ell} H_{\varnothing}>{ }^{\varnothing} E_{t} H_{\varnothing}$, the modulus $E_{\ell} H$ is always less than ${ }^{\varnothing} E_{\ell} H_{\varnothing}$. For instance, with $R_{\varnothing}=0.1 \mathrm{~m}$ and $p_{\max }=600 \mathrm{kPa}$, the difference is $-4.2 \%$. With $R_{\varnothing}=0.15 \mathrm{~m}$ and $p_{\max }=450 \mathrm{kPa}$, the difference is $-4.0 \%$ while with $R_{\varnothing}=0.2 \mathrm{~m}$ and $p_{\max }=300 \mathrm{kPa}$, it is $-4.2 \%$.

- The shear modulus $G_{\ell t} H$ in the pre-stressed reference configuration depends on the ratio product $\frac{L}{L_{\varnothing}} \frac{R}{R_{\varnothing}}$ and it increases almost linearly as a function of pressure $p$ in the range of pressures shown.

Let us quantify the relative difference of the shear modulus when the pressure takes the maximum value in the tube. With $R_{\varnothing}=0.1 \mathrm{~m}$ and $p_{\max }=600 \mathrm{kPa}$, the relative difference is $22.4 \%$. With $R_{\varnothing}=0.15 \mathrm{~m}$ and $p_{\max }=450 \mathrm{kPa}$, the difference is $25.5 \%$ while with $R_{\varnothing}=0.2 \mathrm{~m}$ and $p_{\max }=300 \mathrm{kPa}$, it is $22.4 \%$. These differences are significant.

\section{Orientation 2}

- Figure 6 related to the case of Orientation 2 shows that, contrary to the case of Orientation 1, the modulus $E_{\ell} H$ always increases with the pressure. At the maximal tensile stress that each tube can bear, the relative difference is $15.8 \%$ when $R_{\varnothing}=0.1 \mathrm{~m}, 18 \%$ when $R_{\varnothing}=0.15 \mathrm{~m}$ and $15.8 \%$ when $R_{\varnothing}=0.2 \mathrm{~m}$.

- The modulus $G_{\ell t} H$ also increases with the internal pressure $p$, as in the case of Orientation 1. At the maximal tensile stress that each tube can bear, the relative 


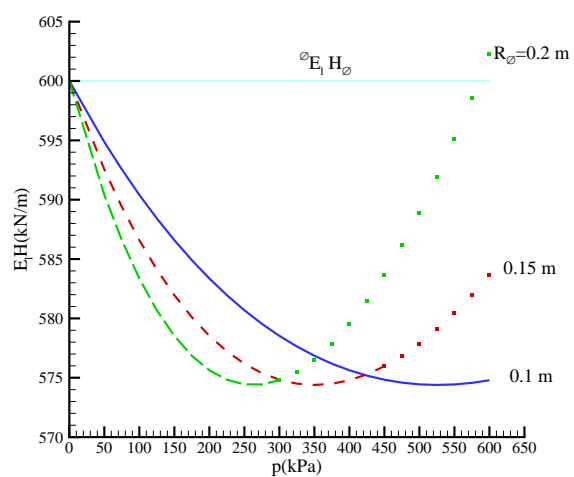

(a) Young's modulus $E_{\ell} H$

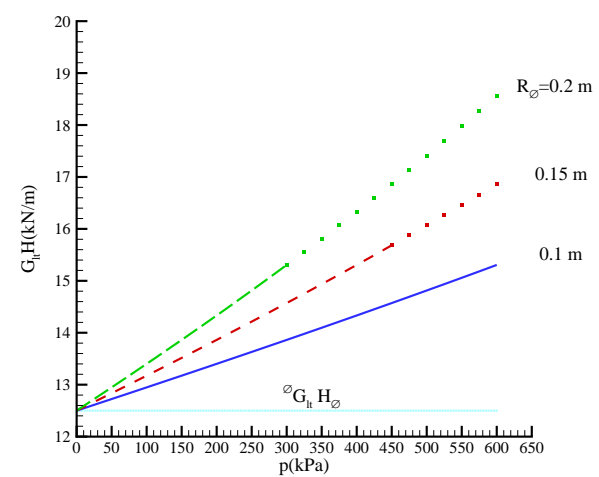

(b) Shear modulus $G_{\ell t} H$

Figure 5: Material coefficients $\left(E_{\ell} H, G_{\ell t} H\right)$ versus the internal pressure - Orientation 1

difference is $17.9 \%$ when $R_{\varnothing}=0.1 \mathrm{~m}, 20.4 \%$ when $R_{\varnothing}=0.15 \mathrm{~m}$ and $17.9 \%$ when $R_{\varnothing}=0.2 m$.

As can be seen, the differences between the moduli in the natural configuration and the pre-stressed reference configuration may be significant.

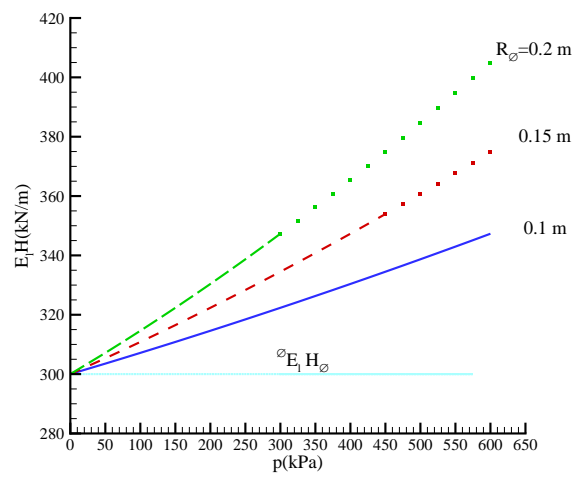

(a) Young's modulus $E_{\ell} H$

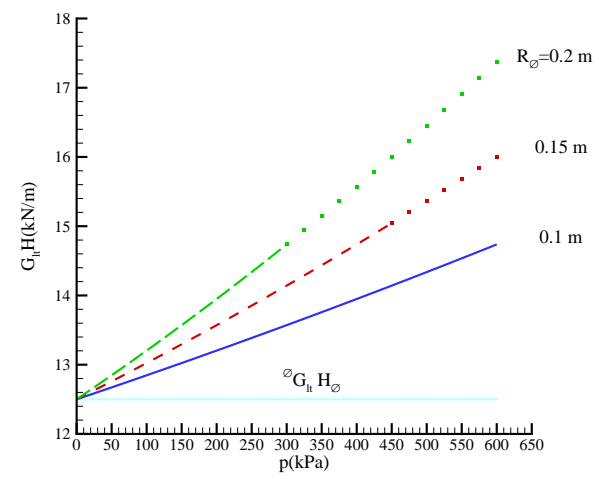

(b) Shear modulus $G_{\ell t} H$

Figure 6: Material coefficients $\left(E_{t} H, G_{\ell t} H\right)$ versus the internal pressure - Orientation 2

The numerical values of the material coefficients $E_{t} H, G_{\ell t} H$ versus the internal pressure are displayed in Table 2. 
Table 2: Geometry and material properties of the tube in the pressurized configuration

Orientation 1

\begin{tabular}{|c|c|c|c|c|c|c|c|}
\hline \multirow[b]{2}{*}{$R_{\varnothing}(m)$} & \multirow{2}{*}{$p(k P a)$} & \multirow{2}{*}{$L(m)$} & \multirow{2}{*}{$R(m)$} & \multirow{2}{*}{$\begin{array}{c}E_{\ell} H \\
(k N / m)\end{array}$} & \multirow{2}{*}{$\begin{array}{c}G_{\ell t} H \\
(k N / m)\end{array}$} & \multirow{2}{*}{\multicolumn{2}{|c|}{ Change (\%) }} \\
\hline & & & & & & & \\
\hline \multirow{12}{*}{0.1} & 50 & 2.5057 & 0.10156 & 594.861 & 12.723 & 1. & 2 \\
\hline & 100 & 2.51196 & 0.10309 & 590.408 & 12.948 & 2. & 4. \\
\hline & 150 & 2.51876 & 0.10461 & 586.591 & 13.174 & 2. & 5. \\
\hline & 200 & 2.52608 & 0.1061 & 583.364 & 13.401 & 3. & 7. \\
\hline & 250 & 2.53391 & 0.10759 & 580.689 & 13.631 & 3. & 9. \\
\hline & 300 & 2.54224 & 0.10906 & 578.529 & 13.862 & 4. & 11. \\
\hline & 350 & 2.55104 & 0.11051 & 576.852 & 14.096 & 4. & 13. \\
\hline & 400 & 2.56031 & 0.11196 & 575.629 & 14.333 & 4. & 15. \\
\hline & 450 & 2.57003 & 0.1134 & 574.834 & 14.572 & 4. & 17. \\
\hline & 500 & 2.58018 & 0.11482 & 574.443 & 14.813 & 4. & 19. \\
\hline & 550 & 2.59077 & 0.11625 & 574.436 & 15.058 & 4. & 21. \\
\hline & 600 & 2.60176 & 0.11766 & 574.791 & 15.306 & 4. & 22. \\
\hline \multirow{12}{*}{0.15} & 50 & 2.50876 & 0.15349 & 592.552 & 12.835 & 1. & 3. \\
\hline & 100 & 2.51876 & 0.15691 & 586.591 & 13.174 & 2. & 5. \\
\hline & 150 & 2.52993 & 0.16027 & 581.96 & 13.516 & 3. & 8. \\
\hline & 200 & 2.54224 & 0.16359 & 578.529 & 13.862 & 4. & 11. \\
\hline & 250 & 2.55562 & 0.16686 & 576.185 & 14.214 & 4. & 14. \\
\hline & 300 & 2.57003 & 0.1701 & 574.834 & 14.572 & 4. & 17. \\
\hline & 350 & 2.58542 & 0.1733 & 574.393 & 14.935 & 4. & 20 . \\
\hline & 400 & 2.60176 & 0.17649 & 574.791 & 15.306 & 4. & 22. \\
\hline & 450 & 2.61901 & 0.17965 & 575.968 & 15.684 & 4. & 26. \\
\hline & 500 & 2.63712 & 0.1828 & 577.871 & 16.069 & 4. & 29. \\
\hline & 550 & 2.65606 & 0.18594 & 580.451 & 16.462 & 3. & 32. \\
\hline & 600 & 2.67581 & 0.18907 & 583.67 & 16.864 & 3. & 35. \\
\hline \multirow{12}{*}{0.2} & 50 & 2.51196 & 0.20618 & 590.408 & 12.948 & 2. & 4. \\
\hline & 100 & 2.52608 & 0.21221 & 583.364 & 13.401 & 3. & 7. \\
\hline & 150 & 2.54224 & 0.21811 & 578.529 & 13.862 & 4. & 11. \\
\hline & 200 & 2.56031 & 0.22392 & 575.629 & 14.333 & 4. & 15. \\
\hline & 250 & 2.58018 & 0.22965 & 574.443 & 14.813 & 4. & 19. \\
\hline & 300 & 2.60176 & 0.23532 & 574.791 & 15.306 & 4. & 22. \\
\hline & 350 & 2.62495 & 0.24094 & 576.524 & 15.811 & 4. & 27. \\
\hline & 400 & 2.64966 & 0.24653 & 579.518 & 16.33 & 3. & 31. \\
\hline & 450 & 2.67581 & 0.25209 & 583.67 & 16.864 & 3. & 35. \\
\hline & 500 & 2.70332 & 0.25764 & 588.894 & 17.412 & 2. & 39. \\
\hline & 550 & 2.73214 & 0.26319 & 595.117 & 17.977 & 1. & 44. \\
\hline & 600 & 2.76219 & 0.26874 & 602.278 & 18.557 & 0.4 & 49. \\
\hline
\end{tabular}


Orientation 2

\begin{tabular}{|c|c|c|c|c|c|c|c|}
\hline \multirow[b]{2}{*}{$R_{\varnothing}(m)$} & \multirow[b]{2}{*}{$p(k P a)$} & \multirow[b]{2}{*}{$L(m)$} & \multirow{2}{*}{$R(m)$} & \multirow{2}{*}{$\begin{array}{c}E_{\ell} H \\
(\mathrm{kN} / \mathrm{m})\end{array}$} & \multirow{2}{*}{$\begin{array}{c}G_{\ell t} H \\
(k N / m)\end{array}$} & \multicolumn{2}{|c|}{ Change (\%) } \\
\hline & & & & & & in $E_{\ell} H$ & in $G_{\ell t} H$ \\
\hline \multirow{12}{*}{0.1} & 50 & 2.51592 & 0.10074 & 303.538 & 12.672 & 1. & 1. \\
\hline & 100 & 2.53203 & 0.10147 & 307.152 & 12.847 & 2. & 3. \\
\hline & 150 & 2.54831 & 0.10222 & 310.84 & 13.024 & 4. & 4. \\
\hline & 200 & 2.56476 & 0.10296 & 314.602 & 13.204 & 5. & 6. \\
\hline & 250 & 2.58137 & 0.10371 & 318.437 & 13.386 & 6. & 7. \\
\hline & 300 & 2.59816 & 0.10447 & 322.345 & 13.571 & 7. & 9. \\
\hline & 350 & 2.6151 & 0.10522 & 326.325 & 13.759 & 9. & 10. \\
\hline & 400 & 2.6322 & 0.10599 & 330.377 & 13.949 & 10. & 12. \\
\hline & 450 & 2.64946 & 0.10675 & 334.501 & 14.142 & 12. & 13. \\
\hline & 500 & 2.66688 & 0.10752 & 338.696 & 14.338 & 13. & 15. \\
\hline & 550 & 2.68444 & 0.1083 & 342.963 & 14.536 & 14. & 16. \\
\hline & 600 & 2.70216 & 0.10908 & 347.301 & 14.737 & 16. & 18. \\
\hline \multirow{12}{*}{0.15} & 50 & 2.52395 & 0.15166 & 305.335 & 12.759 & 2. & 2. \\
\hline & 100 & 2.54831 & 0.15332 & 310.84 & 13.024 & 4. & 4. \\
\hline & 150 & 2.57304 & 0.15501 & 316.51 & 13.295 & 6. & 6. \\
\hline & 200 & 2.59816 & 0.1567 & 322.345 & 13.571 & 7. & 9. \\
\hline & 250 & 2.62363 & 0.15841 & 328.342 & 13.853 & 9. & 11. \\
\hline & 300 & 2.64946 & 0.16013 & 334.501 & 14.142 & 12. & 13. \\
\hline & 350 & 2.67564 & 0.16186 & 340.821 & 14.436 & 14. & 16. \\
\hline & 400 & 2.70216 & 0.16361 & 347.301 & 14.737 & 16. & 18. \\
\hline & 450 & 2.72901 & 0.16538 & 353.941 & 15.044 & 18. & 20. \\
\hline & 500 & 2.75619 & 0.16716 & 360.742 & 15.357 & 20. & 23. \\
\hline & 550 & 2.78369 & 0.16895 & 367.704 & 15.677 & 23. & 25. \\
\hline & 600 & 2.8115 & 0.17076 & 374.826 & 16.003 & 25. & 28. \\
\hline \multirow{12}{*}{0.2} & 50 & 2.53203 & 0.20295 & 307.152 & 12.847 & 2. & 3. \\
\hline & 100 & 2.56476 & 0.20592 & 314.602 & 13.204 & 5. & 6. \\
\hline & 150 & 2.59816 & 0.20893 & 322.345 & 13.571 & 7. & 9. \\
\hline & 200 & 2.6322 & 0.21197 & 330.377 & 13.949 & 10. & 12. \\
\hline & 250 & 2.66688 & 0.21505 & 338.696 & 14.338 & 13. & 15. \\
\hline & 300 & 2.70216 & 0.21815 & 347.301 & 14.737 & 16. & 18. \\
\hline & 350 & 2.73803 & 0.22129 & 356.191 & 15.148 & 19. & 21. \\
\hline & 400 & 2.77448 & 0.22447 & 365.365 & 15.569 & 22. & 25. \\
\hline & 450 & 2.8115 & 0.22767 & 374.826 & 16.003 & 25 . & 28. \\
\hline & 500 & 2.84907 & 0.23092 & 384.574 & 16.448 & 28. & 32. \\
\hline & 550 & 2.88718 & 0.2342 & 394.611 & 16.904 & 32. & 35. \\
\hline & 600 & 2.92582 & 0.23751 & 404.938 & 17.373 & 35 . & 39. \\
\hline
\end{tabular}

The obtained results show that the material coefficients in the pre-stressed reference configuration may notably differ from the material coefficients in the natural reference configuration. For this reason, it is quite essential to use the former coefficients, not the latter, in mechanical problems with pre-stressed reference configuration, as is the case of the membrane tube subjected to internal pressure and bending loads which we are going to investigate.

It is noteworthy that modulus $E_{\ell} H$ may decrease or increase, depending on ${ }^{\varnothing} E_{\ell} H_{\varnothing}>$ ${ }^{\varnothing} E_{t} H_{\varnothing}$ or ${ }^{\varnothing} E_{\ell} H_{\varnothing}<{ }^{\varnothing} E_{t} H_{\varnothing}$. Furthermore, the change in $E_{\ell} H$ and $G_{\ell t} H$ may be more than $30 \%$ in some cases. 


\section{EQUILIBRIUM EQUATIONS FOR THE BENDING OF AN INFLATABLE BEAM}

Let us now pass on to the study of the in-plane bending of the membrane tube inflated at a given pressure $p$. To do this, we shall extend the approach in [10] to the orthotropic case and formulate the problem in some detail since the transition from the isotropic to the orthotropic case is not so straightforward. From this point on, the reference configuration will be the pressurized pre-stressed one $\Omega_{0}$, the final configuration will be the pressurized-and-then-bent configuration $\Omega$. The transition from the former to the latter configuration is represented by the deformation $\boldsymbol{\Phi}$ defined in (1), see Figure 1. This Section briefly recalls - with some improvements - the formulation of the inflatable beam theory by means of the virtual power principle as reported in [10].

Use will be made of the total Lagrangian formulation with the Lagrangian variables defined with respect to the reference configuration $\Omega_{0}$ : $\mathbf{F}$ is the deformation gradient tensor, $\boldsymbol{\Pi}$ (respectively $\boldsymbol{\Sigma}$ ) is the first (respectively second) Piola-Kirchhoff stress tensor, $\rho_{0} \mathbf{f}_{0}$ the force per unit mass in the reference state.

The equilibrium equations are derived from the virtual power principle which stipulates that the following equality holds for any virtual velocity field $\mathbf{V}^{*}$

$$
-\int_{\Omega_{0}}(\mathbf{F} \boldsymbol{\Sigma})^{T}: \operatorname{gradV}^{*} d \Omega_{0}+\int_{\Omega_{0}} \rho_{0} \mathbf{f}_{0} \mathbf{V}^{*} d \Omega_{0}+\int_{\partial \Omega_{0}} \mathbf{T V}^{*} d S_{0}=0
$$

where $\partial \Omega_{0}$ is the boundary of region $\Omega_{0}, \mathbf{T}=\Pi \mathbf{N}$ is the nominal stress vector. Here, for the membrane tube, the virtual power $\int_{\partial \Omega_{0}} \mathbf{T V}^{*} d S_{0}$ of the surface loads includes the contribution of the internal pressure. This contribution writes $\int_{S_{p}} \mathbf{V}^{*} p \mathbf{n} d S$, where $S_{p}$ is the portion of surface on which pressure $p$ is prescribed, in this case $S_{p}$ is the internal tube wall. Since the pressure is a follower load, $S_{p}$ is the current surface (in the deformed configuration) and $\mathbf{n}$ is the normal to the current surface.

\subsection{Kinematics}

The bending problem is conducted considering the tube as a thin-walled beam. In order to account for shear effects, we assume the Timoshenko kinematics for the tube, 
according to which the cross-section remains plane during the deformation but is not perpendicular to the deformed neutral axis.

Let us denote $X$ the reference abscissa of the centroid $\mathbf{G}$ of the cross-section, $\mathbf{U}(X)=(U(X), V(X), 0)$ the displacement vector of $\mathbf{G}$ and $\boldsymbol{\theta}(X)$ the rotation about $\mathbf{e}_{z}$ of the cross-section (all the components are related to the Cartesian basis $\left(\mathbf{e}_{x}, \mathbf{e}_{y}, \mathbf{e}_{z}\right)$, see Figure 7). The reference position of any particle in the cross-section is $\mathbf{X}(X, Y, Z)$, the current position $\mathbf{x}$ of the same particle is then defined from $\mathbf{X}$ by

$$
\begin{aligned}
\mathbf{x}=\mathbf{\Phi}(\mathbf{X}) & =\mathbf{X}+\mathbf{U}(X)+(\mathbf{R}-\mathbf{I}) \cdot \mathbf{G X} \\
& =\left\{\begin{array}{c}
X \\
Y \\
Z
\end{array}\right\}+\left\{\begin{array}{l}
U \\
V \\
0
\end{array}\right\}+\left[\begin{array}{lll}
\cos \theta-1 & -\sin \theta & 0 \\
\sin \theta & \cos \theta-1 & 0 \\
0 & 0 & 0
\end{array}\right] \cdot\left\{\begin{array}{l}
0 \\
Y \\
Z
\end{array}\right\}=\left\{\begin{array}{l}
X+U-Y \sin \theta \\
V+Y \cos \theta \\
Z
\end{array}\right\}
\end{aligned}
$$

where $\mathbf{R}$ et $\mathbf{I}$ are the rotation and identity tensor, respectively; and the components in the column-vectors and matrice are related to the Cartesian basis $\left(\mathbf{e}_{x}, \mathbf{e}_{y}, \mathbf{e}_{z}\right)$.

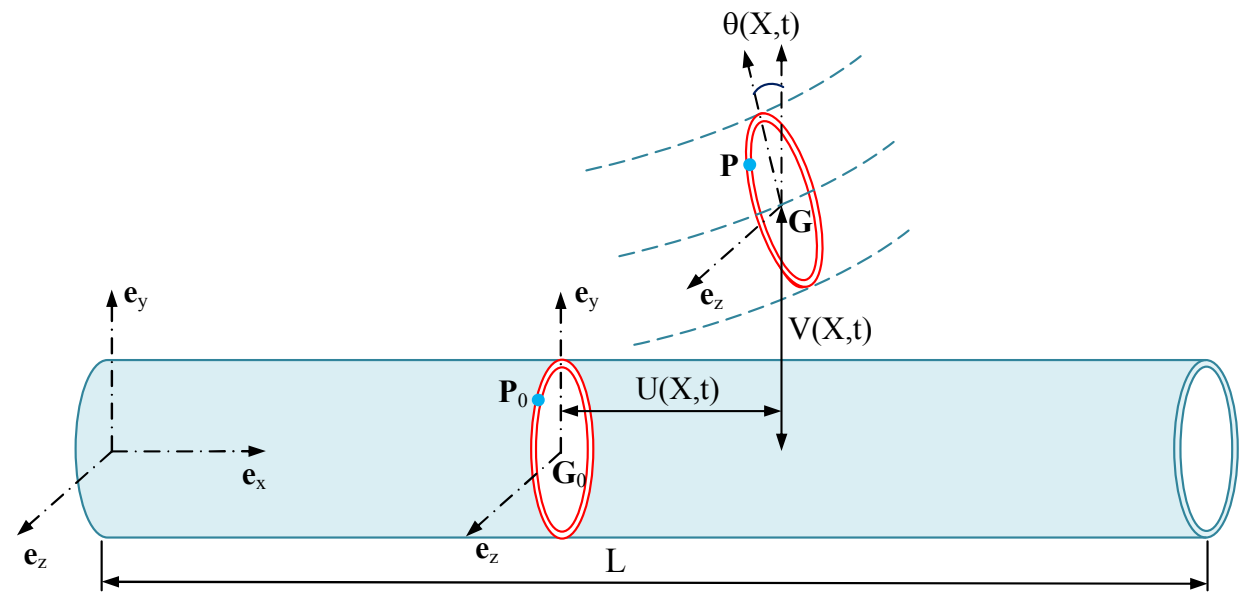

Figure 7: Kinematics of the pressurized membrane tube

There follows the matrix of the deformation gradient tensor $\mathbf{F}$ in the fixed basis $\left(\mathbf{e}_{x}, \mathbf{e}_{y}, \mathbf{e}_{z}\right):$

$$
\operatorname{Mat}\left(\mathbf{F} ; \mathbf{e}_{x}, \mathbf{e}_{y}, \mathbf{e}_{z}\right)=\left[\begin{array}{lcl}
U, X-Y \cos \theta \theta, X+1 & -\sin \theta & 0 \\
V, X-Y \sin \theta \theta, X & \cos \theta & 0 \\
0 & 0 & 1
\end{array}\right]
$$


The components of the Green strain tensor $\mathbf{E}$ in the same basis write are

$$
\begin{aligned}
E_{X X} & =U,_{X}-Y \cos \theta \theta,,_{X}+\frac{1}{2}\left(U,_{X}^{2}+V,_{X}^{2}+Y^{2} \theta_{,}^{2}\right)-\left(U,_{X} \cos \theta \theta,_{X} Y+V,_{X} \sin \theta \theta,,_{X} Y\right) \\
E_{X Y} & =\frac{1}{2}\left[V,,_{X} \cos \theta-(1+U, X) \sin \theta\right] \\
E_{Y Y} & =E_{Z Z}=E_{Y Z}=E_{Z X}=0
\end{aligned}
$$

\subsection{Virtual kinematics}

Let $\mathbf{V}^{*}(\mathbf{G})=\left(U^{*}(X), V^{*}(X), 0\right)$ be the virtual velocity of the centroid $\mathbf{G}$ and $\boldsymbol{\theta}^{*}(\mathbf{G})=$ $\left(0,0, \theta^{*}(X)\right)$ the virtual rotation of the current cross-section. The virtual velocity $\mathbf{V}^{*}(\mathbf{X})$ of a current particle in Equation (26) is chosen as

$\mathbf{V}^{*}(\mathbf{X})=\mathbf{V}^{*}(\mathbf{G})+\boldsymbol{\theta}^{*}(\mathbf{G}) \times \mathbf{g x}=\left\{\begin{array}{l}U^{*} \\ V^{*} \\ 0\end{array}\right\}+\left\{\begin{array}{l}0 \\ 0 \\ \theta^{*}\end{array}\right\} \times\left\{\begin{array}{c}-Y \sin \theta \\ Y \cos \theta \\ Z\end{array}\right\}=\left\{\begin{array}{c}U^{*}-Y \cos \theta \theta^{*} \\ V^{*}-Y \sin \theta \theta^{*} \\ 0 \\ (30)\end{array}\right\}$

It should be noted that, as in the case of rigid body mechanics, the virtual velocity in Equation (30) involves the final position vector $\mathbf{g x}$, not the initial position vector GX. It remains to express $\mathbf{g x}$ in terms of the Lagrangian variables so that one can use the virtual power principle (26) written in Lagrangian form. One has

$$
\mathbf{g} \mathbf{x}=\mathbf{R} \cdot \mathbf{G X}=\left[\begin{array}{ccc}
\cos \theta & -\sin \theta & 0 \\
\sin \theta & \cos \theta & 0 \\
0 & 0 & 1
\end{array}\right] \cdot\left\{\begin{array}{l}
0 \\
Y \\
Z
\end{array}\right\}=\left\{\begin{array}{c}
-Y \sin \theta \\
Y \cos \theta \\
Z
\end{array}\right\}
$$

Therefore

$$
\mathbf{V}^{*}(\mathbf{X})=\left\{\begin{array}{l}
U^{*} \\
V^{*} \\
0
\end{array}\right\}+\left\{\begin{array}{l}
0 \\
0 \\
\theta^{*}
\end{array}\right\} \times\left\{\begin{array}{c}
-Y \sin \theta \\
Y \cos \theta \\
Z
\end{array}\right\}=\left\{\begin{array}{c}
U^{*}-Y \cos \theta \theta^{*} \\
V^{*}-Y \sin \theta \theta^{*} \\
0
\end{array}\right\}
$$

The matrix of tensor $\operatorname{grad}_{X} \mathbf{V}^{*}(\mathbf{X})$ in the Cartesian basis $\left(\mathbf{e}_{x}, \mathbf{e}_{y}, \mathbf{e}_{z}\right)$ is

$\operatorname{Mat}\left(\operatorname{grad}_{X} \mathbf{V}^{*}(\mathbf{X}) ; \mathbf{e}_{x}, \mathbf{e}_{y}, \mathbf{e}_{z}\right)=\left[\begin{array}{ccc}U^{*},{ }_{X}-Y \cos \theta \theta^{*}, X+Y \sin \theta \theta,{ }_{X} \theta^{*} & -\cos \theta \theta^{*} & 0 \\ V^{*},{ }_{X}-Y \sin \theta \theta,{ }_{X}-Y \cos \theta \theta^{*}{ }_{X} \theta^{*} & -\sin \theta \theta^{*} & 0 \\ 0 & 0 & 0\end{array}\right]$

\subsection{Virtual power of the external loadings}

The virtual power of dead loads is calculated in a standard manner. By denoting $p_{X}, p_{Y}$ the distributed loads per unit length along $\mathbf{e}_{x}, \mathbf{e}_{y} ; \mu$ the distributed torque in 
the $\mathbf{e}_{z}$ direction; $X(),. Y(),. \Gamma($.$) the resultant force and the resultant torque at the tips$ $X=0$ et $X=L$, this virtual power is expressed as

$$
\begin{aligned}
W_{\text {dead loads }}^{*}= & \int_{\Omega_{0}} \rho_{0} \mathbf{f}_{0} \mathbf{V}^{*} d \Omega_{0}+\int_{\partial \Omega_{0}} \mathbf{T V}^{*} d S_{0} \\
= & \int_{0}^{L}\left(p_{X} U^{*}+p_{Y} V^{*}+\mu \theta^{*}\right) d X+X(0) U^{*}(0)+Y(0) V^{*}(0)+\Gamma(0) \theta^{*}(0) \\
& +X(L) U^{*}(L)+Y(L) V^{*}(L)+\Gamma(L) \theta^{*}(L)
\end{aligned}
$$

In the above, only the dead loads are taken into account in the nominal stress vector T. As previously said, one also has to consider the virtual power of the internal pressure $p$ :

$$
\begin{aligned}
W_{\text {pressure }}^{*}=\int_{S_{p}} \mathbf{V}^{*} p \mathbf{n} d S= & P \int_{0}^{L}\left\{U^{*} \sin \theta \theta,_{X}-V^{*} \cos \theta \theta,,_{X}+\theta^{*}\left[V,,_{X} \cos \theta-(1+U, X) \sin \theta\right]\right\} d X \\
& +P\left(U^{*} \cos \theta+V^{*} \sin \theta\right) \mid \begin{array}{l}
L \\
0
\end{array}
\end{aligned}
$$

where $P \equiv p \pi R^{2}$ is the pressure resultant over the cross-section in the pre-stressed reference configuration.

\subsection{Virtual power of the internal forces}

The internal virtual power is obtained from Relations (28) and (32):

$$
\begin{aligned}
W_{\text {int }}^{*}= & -\int_{\Omega_{0}}(\mathbf{F} \boldsymbol{\Sigma})^{T}: \mathbf{g r a d V}^{*} d \Omega_{0} \\
= & -\int_{0}^{L}\left\{\left[N(1+U, X)+M \cos \theta \theta,,_{X}-T \sin \theta\right] U^{*},_{X}+\left(N V, X+M \sin \theta \theta,_{X}+T \cos \theta\right) V^{*},_{X}\right. \\
& +\left[-M\left(1+U,_{X}\right) \sin \theta \theta,_{X}+M V,_{X} \cos \theta \theta,_{X}-\left[\left(1+U,,_{X}\right) \cos \theta+V,_{X} \sin \theta\right] T\right] \theta^{*} \\
& \left.+\left[M\left(1+U,_{X}\right) \cos \theta+M V,_{X} \sin \theta+M^{(2)} \theta,_{X}\right] \theta^{*},_{X}\right\} d X
\end{aligned}
$$

In the above equation, the stress resultants $N, M, T, M^{(2)}$ are defined by

$$
N \equiv \int_{S_{0}} \Sigma_{X X} d S_{0} \quad M \equiv-\int_{S_{0}} Y \Sigma_{X X} d S_{0} \quad T \equiv \int_{S_{0}} \Sigma_{X Y} d S_{0} \quad M^{(2)} \equiv \int_{S_{0}} Y^{2} \Sigma_{X X} d S_{0}
$$

where $S_{0}$ is the reference cross-section of the tube. 


\subsection{System of nonlinear equations of the problem}

By inserting Relations (33), (34) and (35) into the virtual power principle (26) and carrying out adequate integrations by parts yield the following equilibrium equations for the pressurized and bent membrane tube:

$$
\begin{aligned}
& -\left[N\left(1+U_{, X}\right)\right]_{, X}-\left(M \cos \theta \theta,_{X}\right),_{X}+(T \sin \theta),_{X}-P \sin \theta \theta, X=p_{X} \\
& -\left(N V_{, X}\right)_{, X}-\left(M \sin \theta \theta,_{X}\right),_{X}-(T \cos \theta)_{, X}+P \cos \theta \theta,_{X}=p_{Y} \\
& -\left[M\left(1+U,,_{X}\right)\right],{ }_{X} \cos \theta-\left(M V,_{X}\right){ }_{X} \sin \theta-T\left(1+U,_{X}\right) \cos \theta \\
& -T V,_{X} \sin \theta-\left(M^{(2)} \theta,_{X}\right),,_{X}-P\left[V,,_{X} \cos \theta-\left(1+U,_{X}\right) \sin \theta\right]=\mu
\end{aligned}
$$

together with the boundary conditions:

$$
\begin{aligned}
& N(0)\left[1+U,_{X}(0)\right]+M(0) \cos \theta(0) \theta,_{X}(0)-T(0) \sin \theta(0)-P \cos \theta(0)=-X(0) \\
& N(L)[1+U, X(L)]+M(L) \cos \theta(L) \theta, X(L)-T(L) \sin \theta(L)-P \cos \theta(L)=+X(L) \\
& N(0) V,_{X}(0)+M(0) \sin \theta(0) \theta, X(0)-T(0) \cos \theta(0)-P \sin \theta(0) \quad=-Y(0) \\
& N(L) V,,_{X}(L)+M(L) \sin \theta(L) \theta, X(L)-T(L) \cos \theta(L)-P \sin \theta(L)=+Y(L) \\
& M(0)\left[1+U,_{X}(0)\right] \cos \theta(0)+M(0) V_{, X}(0) \sin \theta(0)+M^{(2)}(0) \theta_{, X}(0)=-\Gamma(0) \\
& M(L)\left[1+U,_{X}(L)\right] \cos \theta(L)+M(L) V_{X}(L) \sin \theta(L)+M^{(2)}(L) \theta_{, X}(L)=+\Gamma(L)
\end{aligned}
$$

The (through the thickness) stress resultants (36) can be expressed in terms of the displacements $U, V$ and the rotation $\theta$ by means of the constitutive law (24) and the strains (29):

$$
\begin{aligned}
N & =N_{0}+E_{\ell} S_{0}\left[U, X+\frac{1}{2}\left(U,,_{X}^{2}+V,,_{X}^{2}+\frac{I_{0}}{S_{0}} \theta,,_{X}^{2}\right)\right] \\
T & =T_{0}+k G_{\ell t} S_{0}[V, X \cos \theta-(1+U, X) \sin \theta] \\
M & \left.=M_{0}+E_{\ell} I_{0}\left[(1+U, X) \cos \theta+V,,_{X} \sin \theta\right) \theta,,_{X}\right] \\
M^{(2)} & =M_{0}^{(2)}+E_{\ell} I_{0}\left[U,{ }_{X}+\frac{1}{2}\left(U,_{X}^{2}+V,_{X}^{2}+\frac{K_{0}}{I_{0}} \theta,_{X}^{2}\right)\right]
\end{aligned}
$$

where symbol $S_{0}$ for the reference cross-section has been re-used to denote the area of the same section, $I_{0}$ the second moment of area of the cross-section, $k$ is the shear factor (equal to $1 / 2$ in the case in hand of a round tube), $K_{0} \equiv \int_{S_{0}} Y^{4} d S_{0}$ is a coefficient which only depends on the cross-section geometry. Finally, the initial resultant stresses $N_{0}, T_{0}, M_{0}, M_{0}^{(2)}$ are defined in a similar way to (36), involving the pre-stresses $\Sigma_{X X}^{0}$ et $\Sigma_{X Y}^{0}$ appearing in the constitutive law (24):

$$
N_{0} \equiv \int_{S_{0}} \Sigma_{X X}^{0} d S_{0} \quad M_{0} \equiv-\int_{S_{0}} Y \Sigma_{X X}^{0} d S_{0} \quad T_{0} \equiv \int_{S_{0}} \Sigma_{X Y}^{0} d S_{0} \quad M_{0}^{(2)} \equiv \int_{S_{0}} Y^{2} \Sigma_{X X}^{0} d S_{0}
$$

Equations (37) and (39) form a system of 7 nonlinear equations with 7 unknowns $U(X), V(X), \theta(X), N(X), M(X), T(X)$ and $M^{(2)}(X)$. 


\section{LINEARIZED EQUATIONS FOR THE BENDING PROBLEM}

We proceed to linearize the previous equations around the pre-stressed reference configuration, under the following assumptions which are satisfied in practice:

(i) assumption about the order of magnitude of the kinematical variables: $V / L$ and $\theta$ are infinitesimal of order 1 ; and $U / L$ is infinitesimal of order 2;

(ii) assumption about the pre-stresses : $\Sigma_{X X}^{0}=$ const, $\Sigma_{X Y}^{0}=0$.

Assumption (ii) entails firstly $M_{0}=0$ and $T_{0}=0$, and secondly $N_{0}=S_{0} \Sigma_{X X}^{0}, M_{0}^{(2)}=$ $I_{0} \Sigma_{X X}^{0}=\frac{N_{0}}{S_{0}} I_{0}$. The linearized integrated constitutive laws can then be derived from Relations (39):

$$
N=N_{0} \quad T=k G_{\ell t} S_{0}(V, X-\theta) \quad M=E_{\ell} I_{0} \theta, X \quad M^{(2)}=\frac{N_{0}}{S_{0}} I_{0}
$$

In the same time, the linearized equations for the bending of the inflated tube are derived from Relations (37):

$$
\begin{array}{lll}
-N_{0, X} & = & p_{X} \\
-\left(N_{0}+k G_{\ell t} S_{0}\right) V,_{X}^{2}+\left(P+k G_{\ell t} S_{0}\right) \theta, X & = & p_{Y} \\
-\left(E_{\ell}+\frac{N_{0}}{S_{0}}\right) I_{0} \theta,,_{X}^{2}-\left(P+k G_{\ell t} S_{0}\right)(V, X-\theta) & = & \mu
\end{array}
$$

Finally, the linearized boundary conditions result from Relations (38):

$$
\begin{aligned}
& N_{0}(0)-P \quad=-X(0) \\
& N_{0}(L)-P \quad=+X(L) \\
& \left(N_{0}(0)+k G_{\ell t} S_{0}\right) V, X(0)-\left(P+k G_{\ell t} S_{0}\right) \theta(0)=-Y(0) \\
& \left(N_{0}(L)+k G_{\ell t} S_{0}\right) V, X(L)-\left(P+k G_{\ell t} S_{0}\right) \theta(L)=+Y(L) \\
& \left(E_{\ell}+\frac{N_{0}(0)}{S_{0}}\right) I_{0} \theta,,_{X}(0) \quad=-\Gamma(0) \\
& \left(E_{\ell}+\frac{N_{0}(L)}{S_{0}}\right) I_{0} \theta, X(L) \quad=+\Gamma(L)
\end{aligned}
$$

Equation $(42)_{1}$ and the boundary conditions $(43)_{1-2}$ enable one to determine the axial force $N_{0}$ due to the pre-stress. Once $N_{0}$ is known, Equations $(42)_{2-3}$ and the boundary conditions (43) $)_{3-6}$ allow to obtain the deflection $V$ and the rotation $\theta$. 


\section{APPLICATION TO THE BENDING PROBLEM OF A CANTILEVER PRES- SURIZED MEMBRANE TUBE}

The results obtained in the previous Section will now be applied to the bending of a cantilever pressurized membrane tube. In the pre-stressed reference configuration, the tube is subjected to an internal pressure $p$, its geometry is a closed axisymmetric cylindrical tube, of length $L$, radius $R$ and thickness $H$, see Figure 8 . The tube is clamped at the end $X=0$ and free at the other end $X=L$. After the pressurization, a force $F \mathbf{e}_{y}$ is applied at the end $X=L$.

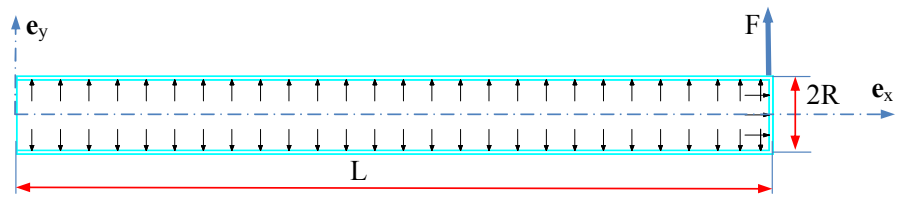

Figure 8: Cantilever pressurized membrane tube subjected to a bending loading

From Equations (42) $)_{1}$ and $(43)_{1-2}$, one gets $N_{0}(X)=P$. The static boundary conditions in terms of $V$ and $\theta$ result from (43) $3-6$ :

$$
\left(P+k G_{\ell t} S_{0}\right)(V, X(L)-\theta(L))=F \quad \theta, X(L)=0
$$

Solving (42) $)_{2-3}$ and (44) under the kinematic boundary conditions $V(0)=\theta(0)=0$ leads to the deflection and the rotation:

$$
\begin{aligned}
& V(X)=\frac{F}{\left(E_{\ell}+\frac{P}{S_{0}}\right) I_{0}}\left(\frac{L X^{2}}{2}-\frac{X^{3}}{6}\right)+\frac{F X}{P+k G_{\ell t} S_{0}} \\
& \theta(X)=\frac{F}{\left(E_{\ell}+\frac{P}{S_{0}}\right) I_{0}}\left(L X-\frac{X^{2}}{2}\right)
\end{aligned}
$$

These relations clearly show the significant role of the internal pressure $p-v i a$ the pressure resultant $P \equiv p \pi R^{2}$ - in the bending and shear stiffnesses of the beam. Equations (45) slightly differ from those given in [12]. With our notations, the modulus ${ }^{\varnothing} \bar{E}_{\ell}=\frac{{ }^{\varnothing} E_{\ell}}{1-{ }^{\varnothing} v_{\ell t} v_{t \ell}}$ in [12] is replaced here by $E_{\ell}$ and the term $\frac{1}{2} k^{\varnothing} G_{\ell t} S_{0}$ in [12] is replaced here by $k G_{\ell t} S_{0}$. 


\section{Influence of the internal pressure on the stiffness moduli}

Relations (45) show that the internal pressure modifies the shear stiffness $k G_{\ell t} S_{0}$, as is the case in Fichter's formulas [6] for the isotropic beam. The pressure also modifies the bending stiffness $E_{\ell} I_{0}$, which extends the result proven in [10] for the isotropic beam again. The formulation of the orthotropic beam may seem elaborate, but the final result is rather intuitive: the transition from the isotropic to the orthotropic case amounts to replacing the Young's modulus $E$ by $E_{\ell}$ and the shear modulus $G$ by $G_{\ell t}$.

In order to quantify the impact of the pressure on the stiffness moduli, let us recast the bending stiffness as

$$
\left(E_{\ell}+\frac{P}{S_{0}}\right) I_{0}=\left(E_{\ell}+\frac{p \pi R^{2}}{2 \pi R H}\right) \pi R^{3} H=\left(E_{\ell} H+\frac{p R}{2}\right) \pi R^{3}
$$

Thus, the change in the Young's modulus due to the pressure is expressed by the correction term $p R / 2$. Likewise, the shear stiffness can be rewritten as

$$
P+k G_{\ell t} S_{0}=p \pi R^{2}+k G_{\ell t} 2 \pi R H=2 \pi R k\left(G_{\ell t} H+\frac{p R}{2 k}\right)
$$

which shows that the change in the shear modulus $G_{\ell t} H$ is equal to $p R$, by taking $k=0.5$ (value for thin tubes).

Consider again the two beams corresponding to two different rolling directions of the orthotropic membrane, denoted Orientation 1 and Orientation 2, with the numerical data given in Table 1. By using the values in Table 2, one can compute the change of the stiffness moduli due to the internal pressure. Table 3 displays the results obtained with pressure $p$ equal to $300 \mathrm{kPa}$.

The shear modulus is more affected by the internal pressure, since it is smaller than the Young's modulus (as is usually the case with fabrics). However, it is found that the change in the Young's modulus - with and without the pressure correction term - is not always negligible, it can be either small (3\%) or significant (9\%). The herein proposed beam theory enables one to precisely compute the stiffness change due to the pressure and decide when some term or other can be neglected.

Influence of the internal pressure on the deflections

Let us now examine the influence of the internal pressure on the beam deflections. For the cantilever beam of interest, the tip deflection $V(L)$ given by (45) can be split 
Table 3: Influence of the internal pressure on the stiffness moduli $E_{\ell} H$ and $G_{\ell t} H$ pour $p=300 k P a$

\section{Orientation 1}

\begin{tabular}{|l|c|c|c|c|c|c|c|}
\multicolumn{8}{c}{${ }^{\varnothing} E_{\ell} H_{\varnothing}=600 \mathrm{kN} / \mathrm{m},{ }^{\varnothing} E_{t} H_{\varnothing}=300 \mathrm{kN} / \mathrm{m},{ }^{\varnothing} G_{\ell t} H_{\varnothing}=12.5 \mathrm{kN} / \mathrm{m},{ }^{\varnothing} v_{\ell t}=0.24$} \\
\hline \multirow{2}{*}{$R_{\varnothing}(m)$} & $R(m)$ & $\begin{array}{c}E_{\ell} H \\
(\mathrm{kN} / \mathrm{m})\end{array}$ & $\begin{array}{c}E_{\ell} H+\frac{p R}{2} \\
(\mathrm{kN} / \mathrm{m})\end{array}$ & $\begin{array}{c}G_{\ell t} H \\
(\mathrm{kN} / \mathrm{m})\end{array}$ & $\begin{array}{c}G_{\ell t} H+p R \\
(\mathrm{kN} / \mathrm{m})\end{array}$ & \multicolumn{2}{c|}{ Change $(\%)$} \\
\hline 0.1 & 0.1091 & 578.53 & 594.89 & 13.86 & 46.58 & $E_{\ell} H+\frac{p R}{2}$ & in $G_{\ell t} H+p R$ \\
0.15 & 0.1701 & 574.83 & 600.35 & 14.57 & 65.60 & -4. & -70. \\
0.3 & 0.2353 & 574.79 & 610.10 & 15.31 & 85.90 & -6. & -78. \\
\hline
\end{tabular}

Orientation 2

\begin{tabular}{|l|c|c|c|c|c|c|c|}
\multicolumn{8}{c}{$E_{\ell} H_{\varnothing}=300 \mathrm{kN} / \mathrm{m},{ }^{\varnothing} E_{t} H_{\varnothing}=600 \mathrm{kN} / \mathrm{m},{ }^{\varnothing} G_{\ell t} H_{\varnothing}=12.5 \mathrm{kN} / \mathrm{m},{ }^{\varnothing} v_{\ell t}=0.12$} \\
\hline \multirow{2}{*}{$R_{\varnothing}(m)$} & $R(m)$ & $\begin{array}{c}E_{\ell} H \\
(\mathrm{kN} / \mathrm{m})\end{array}$ & $\begin{array}{c}E_{\ell} H+\frac{p R}{2} \\
(\mathrm{kN} / \mathrm{m})\end{array}$ & $\begin{array}{c}G_{\ell t} H \\
(\mathrm{kN} / \mathrm{m})\end{array}$ & $\begin{array}{c}G_{\ell t} H+p R \\
(\mathrm{kN} / \mathrm{m})\end{array}$ & \multicolumn{2}{|c|}{ Change $(\%)$} \\
\hline 0.1 & 0.1045 & 322.35 & 338.01 & 13.57 & 44.91 & -5. & -70. \\
0.15 & 0.1601 & 334.50 & 358.52 & 14.14 & 62.18 & -7. & -77. \\
0.3 & 0.2182 & 347.30 & 380.02 & 14.74 & 80.18 & -9. & -82. \\
\hline
\end{tabular}

into two parts, the bending component denoted $V_{B}(L)$ and the shear component $V_{S}(L)$ as follows :

$$
V(L)=\frac{F L^{3}}{3\left(E_{\ell}+\frac{P}{S_{0}}\right) I_{0}}+\frac{F L}{P+k G_{\ell t} S_{0}}=V_{B}(L)+V_{S}(L)
$$

The numerical computations are performed using the data in Table 2 again. The tip force $F$ is taken equal to $1 N$. For the considered three geometries (natural radius $R_{\varnothing}=0.1,0.15$ and $0.2 m$ ), the internal pressure is given its maximal value 600,450 and $300 \mathrm{kPa}$, respectively (see these values in Section 5).

Columns $\% V_{B}(L)$ and $\% V_{S}(L)$ in Table 4 give the part of the bending deflection in comparison with the part of the shear deflection. As is the case of the classical beam theory, the former is always preponderant compared to the latter.

In view of Table 3, one may think that it is possible to disregard the pressure correction in the bending stiffness $E_{\ell} H+\frac{p R}{2}$. In actual fact, since the bending deflection is much greater than the shear deflection, it turns out that an error in the bending stiffness entails an important error in the deflection $V(L)$. To see this, let us denote $V^{(1)}(L)$ (resp. $\left.V^{(2)}(L)\right)$ the tip deflection obtained by omitting the pressure contribution in $E_{\ell} H+\frac{p R}{2}$ 
Table 4: Influence of the internal pressure on the deflection in a cantilever beam, $F=1 \mathrm{~N}$

Orientation 1

\begin{tabular}{|l|c|c|c||c|c|}
\hline $\begin{array}{l}R_{\varnothing} \\
(m)\end{array}$ & $\begin{array}{c}\mathrm{p} \\
(\mathrm{kPa})\end{array}$ & $\% V_{B}(L)$ & $\% V_{S}(L)$ & $\begin{array}{c}\text { Difference (\%) when using } \\
V^{(1)}(L) \text { defined by }(49)\end{array}$ & $\begin{array}{c}\text { Difference (\%) when using } \\
V^{(2)}(L) \text { defined by }(50)\end{array}$ \\
\hline 0.1 & 600 & 96. & 4. & 6. & 19. \\
0.15 & 450 & 92. & 8. & 6. & 43. \\
0.3 & 300 & 85. & 15. & 5. & 68. \\
\hline
\end{tabular}

Orientation 2

\begin{tabular}{|l|c|c|c||c|c|}
\hline $\begin{array}{l}R_{\varnothing} \\
(m)\end{array}$ & $\begin{array}{c}\mathrm{p} \\
(\mathrm{kPa})\end{array}$ & $\% V_{B}(L)$ & $\% V_{S}(L)$ & $\begin{array}{c}\text { Difference }(\%) \text { when using } \\
V^{(1)}(L) \text { defined by }(49)\end{array}$ & $\begin{array}{c}\text { Difference }(\%) \text { when using } \\
V^{(2)}(L) \text { defined by }(50)\end{array}$ \\
\hline 0.1 & 600 & 98. & 2. & 9. & 10. \\
0.15 & 450 & 95. & 5. & 10. & 23. \\
0.3 & 300 & 92. & 8. & 9. & 38. \\
\hline
\end{tabular}

(resp. in $\left.G_{\ell t} H+p R\right)$ :

$$
\begin{aligned}
V^{(1)}(L) & =\frac{F L^{3}}{3 E_{\ell} I_{0}}+\frac{F L}{P+k G_{\ell t} S_{0}} \\
V^{(2)}(L) & =\frac{F L^{3}}{3\left(E_{\ell}+\frac{P}{S_{0}}\right) I_{0}}+\frac{F L}{k G_{\ell t} S_{0}}
\end{aligned}
$$

The last two columns of Table 4 show the errors made when using either $V^{(1)}(L)$ or $V^{(2)}(L)$ instead of deflection $V(L)$ in (48). As expected from Table 3, neglecting $P$ in $P+k G_{\ell t} S_{0}$ may lead to large errors. On the other hand, neglecting $P$ in $E_{\ell}+\frac{P}{S_{0}}$ may also give rise to significant errors, up to $10 \%$ with Orientation 2 and $6 \%$ with Orientation 1.

The proposed formulation makes it possible to quantify the role of different quantities in the response of the beam. In the next section, the validity of the analytical relations (45) will be checked by comparison with finite element computations using the membrane elements. In this respect, it should be emphasized that the point of view as well as the material coefficients to be used are different according to the chosen approach :

- When using the membrane finite element computations, the material input data are coefficients ${ }^{\varnothing} E_{\ell} H_{\varnothing},{ }^{\varnothing} E_{t} H_{\varnothing},{ }^{\varnothing} G_{\ell t} H_{\varnothing}$ and ${ }^{\varnothing} v_{\ell t}$, all defined on the natural state, which is the reference state. The first computational step is the inflation of the membrane tube, the second step is the bending of the inflatable beam, see 
Figure 1.

- When using Relations (45), the material input data are $E_{\ell} H$ and $G_{\ell t} H$, which should be derived from the natural geometry and the applied pressure by means of Relations (25). The reference state is the pressurized one and the bending is the only stage to be considered in Figure 1.

Range of validity of the theory

The proposed theory is valid as long as no wrinkles appear in the membrane. When transforming this into the condition that the principal stresses at any point of the membrane are non-negative, one finds that the force $F$ applied must be less than a value $F_{c}$ referred to as the wrinkling force:

$$
F_{c}=\frac{\pi R^{3} p}{2 L}
$$

This relation is the same as that given in [10] for an isotropic membrane. Here, the wrinkling force depends on the internal pressure $p$, and also on the geometry of the tube and the material properties in the natural configuration, via dimensions $R, L$, see (25).

\section{VERIFICATION OF THE BENDING THEORY BY FINITE ELEMENT COM- PUTATIONS}

In order to assess the proposed bending theory, the analytical results (45) will be compared with those obtained by membrane finite element computations. Here, the computations are carried out using an in-house finite element code developed for nonlinear structural mechanics analysis.

In the finite element approach, the inflated beam is formulated as a three-dimensional nonlinear elasticity problem with the reference configuration taken equal to the natural one. The weak form employed is the virtual power principle (26) and the chosen approach is the total Lagrangian formulation. The beam is modeled as a membrane tube and discretized using membrane finite elements which are by definition elements 
without bending stiffness and satisfying the plane-stress assumption. The spatial discretization by finite elements of the weak form leads to a system of nonlinear algebraic equations which can be solved by the iterative Newton scheme. The tangent stiffness matrix is the sum of the standard stiffness due to the internal stresses and the stiffness due to the pressure which is a follower load.

It should be emphasized that the bending theory proposed in this work and the membrane finite element approach used here are quite distinct, especially on the two following points:

- the bending theory has been constructed using the Timoshenko kinematics whereas the membrane finite element is derived from the membrane kinematics, of the shell type but without bending stiffness;

- in the bending theory, the reference configuration is the pressurized pre-stressed configuration. In contrast, in the finite element approach the reference configuration is natural.

The geometry of the tube and the elastic properties of the membrane in the natural state are given in Table 1.

The analytical expressions (8)-(9), (25) and (45) has been proven assuming that the tube remains cylindrical when inflated. Here, in the finite element computations, one end of the tube is closed by an isotropic membrane and the consequence of this is that when the pressure is prescribed, the tube is not quite cylindrical at the closed end. In order to avoid the perturbations due to the bulging end, we consider a tube a little longer than the theoretical natural length $L_{\varnothing}$, as shown in Figure 9. Provided that the supplementary length is enough, the portion of the tube between $X_{\varnothing}=0$ and $X_{\varnothing}=L_{\varnothing}$ remains practically cylindrical in the deformed state. All the deflections and rotations displayed are in the cross-section $X_{\varnothing}=L_{\varnothing}$.

Concerning the boundary conditions, the axial displacement of all nodes in the cross-section $X_{\varnothing}=0$ is kept to zero whereas the displacements of nodes $(A, B, C, D)$ in the plane $\left(\mathbf{e}_{y}, \mathbf{e}_{z}\right)$ are left free along the directions indicated in Figure 9 so as to preserve symmetry and prevent rigid body motions. 


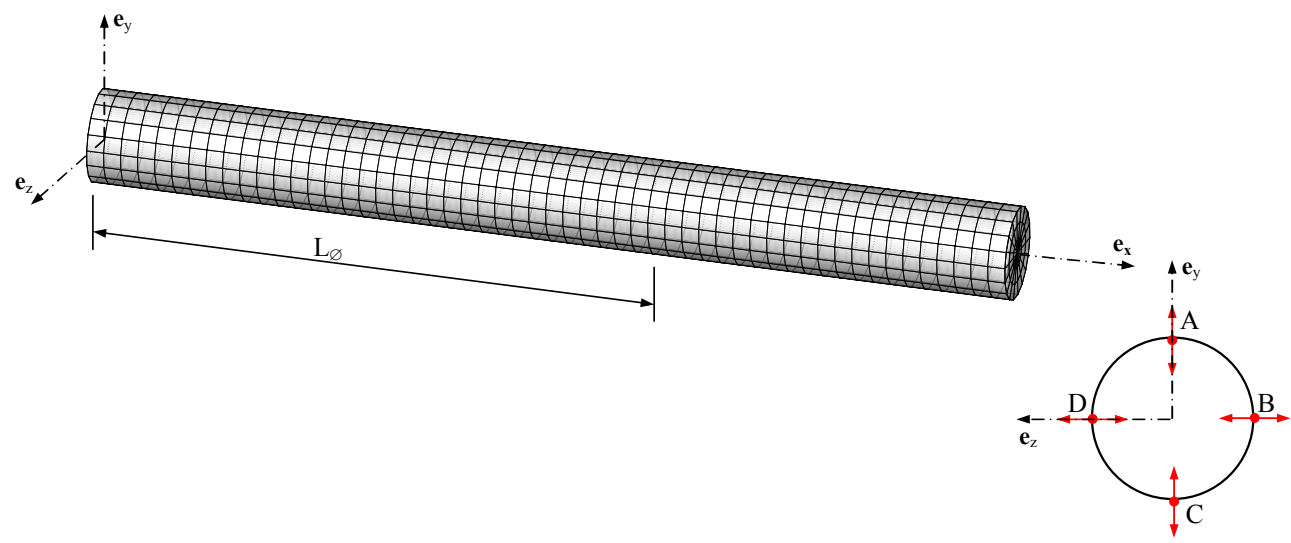

Figure 9: (a) Mesh of the tube with membrane finite elements; (b) Points $A, B, C, D$ on the cross-section $X_{\varnothing}=0$ and the degrees of freedom which are left unprescribed

The internal pressure $p$ is applied progressively. At the first increment, the stiffness matrix is singular as is the case of any non-prestressed membrane structure, thus a small fictitious pre-stress needs to be added to achieve convergence. This pre-stress is immediately removed in the subsequent increments. Once the internal pressure $p$ has reached its prescribed value, the transverse load $F$ is applied on the end $X_{\varnothing}=L_{\varnothing}$. Recall that the membrane finite element code is based on a large deformation formulation and it provides results in the nonlinear framework. The force $F$ applied should not be too large so as to remain in the small deformation context around the pressurized reference configuration and to make it possible to compare the finite element results with the results (45) from the linearized theory. Tests conducted with the membrane finite element code reveal that $F$ can be given the value $1 N$ in order to stay in the linear range, value which is also less than the wrinkling force $F_{c}$ in (51).

The mesh contains 3217 nodes and 1072 membrane elements (Figure 9), which proves to be sufficient for the numerical purposes since in the case $R_{\varnothing}=0.2 m, p=$ $300 \mathrm{kPa}$ for instance, a finer mesh with 6643 nodes gives an identical tip deflection within $0.03 \%$ and an identical tip rotation within $0.02 \%$.

Figures 10 and 11 display the deflection and rotation at the cross-section $X_{\varnothing}=L_{\varnothing}$ versus the internal pressure, for the three tubes of different natural radii $R_{\varnothing}$, and for 
the two orientations no.1 and no.2 (see Table 1). The results obtained from the theory of beam bending (45) will be referred to as analytical solutions, as opposed to the finite element solutions. The curve portions behind the vertical line correspond to the pressures which exceed the maximal admissible pressure in the tube. Logically, comparisons between numerical results should be made with pressures less than the maximal admissible pressure in each tube.

In all cases, the analytical results (45) are found to be in very good agreement with the results from the membrane finite elements. The maximal difference in the deflection is $1.6 \%$ and the difference in the rotation is $2.9 \%$ with Orientation 1 (resp. $1.6 \%$ and $1.8 \%$ with Orientation 2).

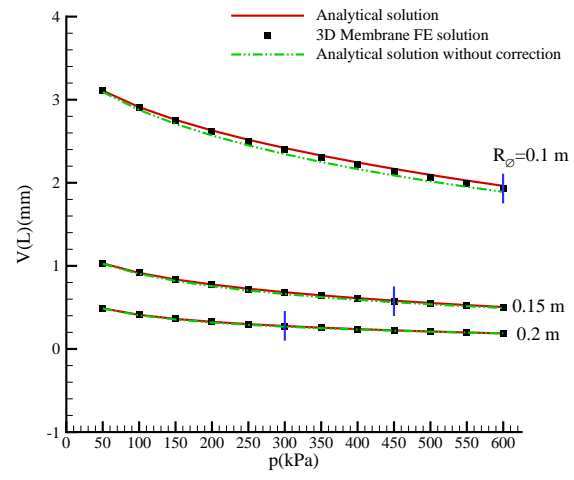

(a) Deflection

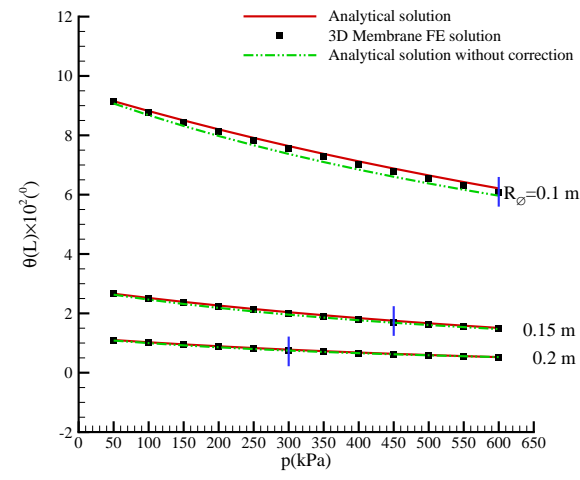

(b) Rotation

Figure 10: Deflection $V(L)$ and rotation $\theta(L)$ of the cross-section at the tip of the tube versus internal pressure $p$ - Orientation 1

REMARK. The material coefficients $E_{\ell} H$ and $G_{\ell t} H$ involved in the analytical solution (45) are related to the pressurized reference configuration and must be computed from coefficients ${ }^{\varnothing} E_{\ell} H_{\varnothing}$ and ${ }^{\varnothing} G_{\ell t} H_{\varnothing}$ by means of Relations (25). What happens if ${ }^{\varnothing} E_{\ell} H_{\varnothing}$ and ${ }^{\varnothing} G_{\ell t} H_{\varnothing}$ are used in (45) instead of $E_{\ell} H$ and $G_{\ell t} H$, in other words what happens if one does not take account of the fact that the material coefficients vary with the pressure? 


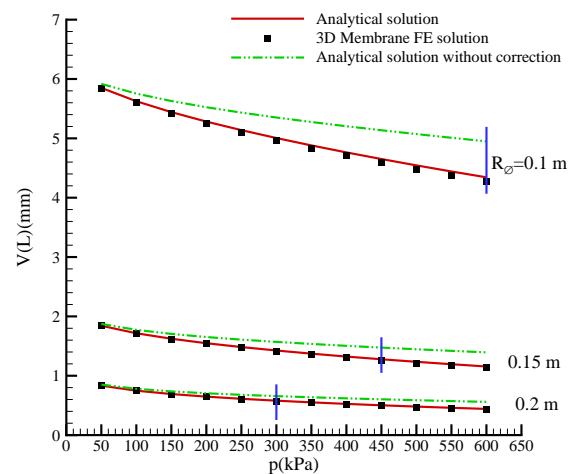

(a) Deflection

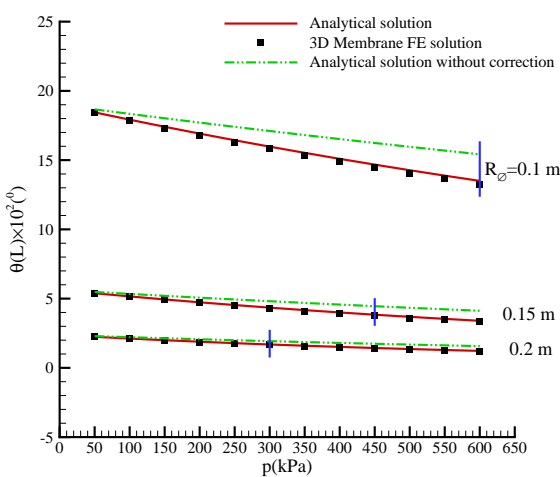

(b) Rotation

Figure 11: Deflection $V(L)$ and rotation $\theta(L)$ of the cross-section at the tip of the versus internal pressure $p$ - Orientation 2

As seen in Section 5.2, in the Orientation 1 case ${ }^{\varnothing} E_{\ell} H_{\varnothing}$ is slightly larger than $E_{\ell} H$ while ${ }^{\varnothing} G_{\ell t} H_{\varnothing}$ is less than $G_{\ell t} H$. If these two coefficients ${ }^{\varnothing} E_{\ell} H_{\varnothing}$ and ${ }^{\varnothing} G_{\ell t} H_{\varnothing}$ are used in (45), the deflection and rotation obtained at the cross-section $X=L$ are slightly less than those given by the analytical solution. Compared with the results obtained by the membrane finite elements, the maximal differences in deflection and rotation are $2.5 \%$ and $2.7 \%$. The results obtained this way are referred to as Analytical solutions without correction in Figures 10 and 11.

On the other hand, in the Orientation 2 case, the material coefficients in the natural configuration ${ }^{\varnothing} E_{t} H_{\varnothing}$ and ${ }^{\varnothing} G_{\ell t} H_{\varnothing}$ are both less than their counterparts in the prestressed reference configuration. The deflection and rotation obtained without correction are then far above those of the analytical solution. In comparison with the results obtained by the membrane finite elements, at the maximum working pressure of the tube and with $R_{\varnothing}=0.1 \mathrm{~m}$, the difference is $15.8 \%$ in deflection and $16.3 \%$ in rotation. With $R_{\varnothing}=0.15 m$, the difference is $17.2 \%$ in deflection and $17.9 \%$ in rotation. Finally, with $R_{\varnothing}=0.2 m$ these differences are $14.6 \%$ and $15.6 \%$

The comparisons above show that it is necessary to use the material coefficients 
related to the pre-stressed reference configuration, not the natural configuration, when computing the bending problem. The very good accordance between the analytical results (45) and the membrane finite element results prove the validity of the proposed theory.

\section{CONCLUSIONS}

The bending of an inflatable beam made of an orthotropic membrane has been investigated in the total Lagrangian framework. The following results have been obtained:

(i) The material coefficients to be used in the analysis take different values, depending on whether they are related to the natural or the pre-stressed configuration. The relationships (25) between the material coefficients related to the two configurations have been established. They clearly show how the change of the tube geometry from the natural to the pre-stressed state comes into play.

(ii) A nonlinear system of equations, Equations (37) and (39), has been obtained, showing the significant role of the internal pressure in the response of inflatable beams.

(iii) Eventually, linearization of the above nonlinear system around the pres-stressed reference configuration has led to linear Equations (42)-(43), which enable one to determine the deflection and the cross-section rotation of the beam. With all these results, the proposed formulation can be considered as giving a new and comprehensive tool for handling orthotropic inflatable beams. As an application, the analytical relations (45) have been derived for a cantilever beam and numerical computations have been carried on this example in order to show how the internal pressure modifies the flexural and shear stiffnesses on the one hand, and the flexural and shear deflections on the other hand.

(iv) The numerical results for different tube geometries, materials and internal pressures have been found to agree very well with the membrane finite element results obtained from an independent code. 


\section{References}

[1] R.L. Comer and S. Levy. Deflections of an inflated circular cylindrical cantilever beam. AIAA Journal, 1(7):1652-1655, 1963.

[2] J.P.H. Webber. Deflections of inflated cylindrical cantilever beams subjected to bending and torsion. Aeronautical Journal, 1020:306-12, 1982.

[3] J.A Main, S.W Peterson, and AM Strauss. Load-deflection behaviour of spacebased inflatable fabric beams. Journal of Aerospace Engineering, 7(2):225-38, 1994.

[4] J.A. Main, S.W. Peterson, and A.M. Strauss. Beam-type bending of space-based membrane structures. Journal of Aerospace Engineering, 8(2):120-125, 1995.

[5] J.D. Suhey, N.H. Kim, and C. Niezrecki. Numerical modeling and design of inflatable structures-application to open-ocean-aquaculture cages. Aquacultural Engineering, 33:285-303, 2005.

[6] W.B. Fichter. A theory for inflated thin-wall cylindrical beams. NASA Technical Note, NASA TND-3466, 1966.

[7] E.C. Steeves. A linear analysis of the deformation of pressure stabilized beams. Technical Report 75-47-AMEL, US Army Natick Laboratories, 1975.

[8] C. Wielgosz and J.C. Thomas. Deflection of inflatable fabric panels at high pressure. Thin-Walled Structures, 40:523-536, 2002.

[9] C. Wielgosz and J.C. Thomas. Deflections of highly inflated fabric tubes. ThinWalled Structures, 42:1049-1066, 2004.

[10] A. Le van and C. Wielgosz. Bending and buckling of inflatable beams: some new theoretical results. Thin-Walled Structures, 43:1166-1187, 2005.

[11] W.G. Davids and H. Zhang. Beam finite-element analysis of pressurized fabric beam-columns. Engineering Structures, 30:1969-80, 2008. 
[12] K.L. Apedo, S. Ronel, E. Jacquelin, M. Massenzio, and A. Bennani. Theoretical analysis of inflatable beams made from orthotropic fabric. Thin-Walled Structures, 47:1507-1522, 2009.

[13] T.T. Nguyen, S. Ronel, M. Massenzio, K.L. Apedo, and E. Jacquelin. Analytical buckling analysis of an inflatable beam made of orthotropic technical textiles. Thin-Walled Structures, 51:186-200, 2012.

[14] P.V. Cavallaro, M.E. Jonhson, and A.M. Sadegh. Mechanics of plain-woven fabrics for inflated structures. Composite structures, 61:375-393, 2003.

[15] A.W. Turner. Experimental test methods for inflatable fabric beams. Master's thesis, University of Maine, 2006.

[16] J.P Kabche, M.L. Peterson, and W. G. Davids. Effect of inflation pressure on the constitutive response of coated woven fabrics used in airbeams. Composites: Part $B, 42: 526-537,2011$.

[17] J.C. Thomas and A. Le van. An exact solution for inflated orthotropic membrane tubes. Thin-Walled Structures, 67:116-120, 2013.

[18] E.N. Dvorkin and M.B. Goldschmit. Nonlinear Continua. Springer, 2006. 